\title{
Moduli Spaces of Isoperiodic Forms on Riemann Surfaces
}

\section{Citation}

McMullen, Curtis T. 2012. "Moduli Spaces of Isoperiodic Forms on Riemann Surfaces." Working paper, Department of Mathematics, Harvard University.

\section{Permanent link}

http://nrs.harvard.edu/urn-3:HUL.InstRepos:11880197

\section{Terms of Use}

This article was downloaded from Harvard University's DASH repository, and is made available under the terms and conditions applicable to Open Access Policy Articles, as set forth at http:// nrs.harvard.edu/urn-3:HUL.InstRepos:dash.current.terms-of-use\#OAP

\section{Share Your Story}

The Harvard community has made this article openly available.

Please share how this access benefits you. Submit a story.

\section{Accessibility}




\title{
Moduli spaces of isoperiodic forms on Riemann surfaces
}

\author{
Curtis T. McMullen
}

6 May 2012

\begin{abstract}
This paper describes the intrinsic geometry of a leaf $\mathcal{A}(L)$ of the absolute period foliation of the Hodge bundle $\Omega \overline{\mathcal{M}}_{g}$ : its singular Euclidean structure, its natural foliations and its discretized Teichmüller dynamics. We establish metric completeness of $\mathcal{A}(L)$ for general $g$, and then turn to a study of the case $g=2$. In this case the Euclidean structure comes from a canonical meromorphic quadratic differential on $\mathcal{A}(L) \cong \mathbb{H}$, whose zeros, poles and exotic trajectories are analyzed in detail.
\end{abstract}

\section{Contents}

1 Introduction . . . . . . . . . . . . . . . . 2

2 Slices of Schottky space . . . . . . . . . . . . . . 12

3 Genus two . . . . . . . . . . . . . . . 17

4 Zero flux ........................ 19

$5 \quad$ Hilbert modular surfaces . . . . . . . . . . . . . . 27

6 Dynamics of $\mathrm{SL}_{2}\left(\mathcal{O}_{D}\right) \ldots \ldots \ldots \ldots . \ldots \ldots$

7 Fixed points and pseudo-Anosov maps . . . . . . . . 36

8 The case $D=5 \ldots \ldots \ldots \ldots \ldots$. . . . . . . . . . . . . . . . . . . .

9 A guide to fake pentagons . . . . . . . . . . . . . 43

10 Class numbers . . . . . . . . . . . . . . . . . . . 44

11 Snow falling on cedars . . . . . . . . . . . . . . 48

Research supported in part by the Humboldt Foundation, the MPI and the NSF. Revised 9 December 2013. 2000 Mathematics Subject Classification: 30F30. 


\section{Introduction}

A compact Riemann surface $X$ of genus $g \geq 2$ acquires a geometric character when it is equipped with a holomorphic 1-form $\omega \in \Omega(X)$. For example, such a form determines a Euclidean metric on $X$ (with singularities at the zeros of $\omega$ ), and a foliation $\mathcal{F}(\omega)$ by horizontal geodesics (provided $\omega \neq 0$ ).

The moduli space of holomorphic 1-forms

$$
\Omega \mathcal{M}_{g} \rightarrow \mathcal{M}_{g}
$$

which classifies pairs $(X, \omega)$ as above, similarly exhibits features not present in the underlying moduli space of curves. For example, $\Omega \mathcal{M}_{g}$ carries a stratified linear structure and a natural action of $\mathrm{SL}_{2}(\mathbb{R})$.

In this paper we study the global geometry of the moduli space of forms $(X, \omega)$ with fixed absolute periods. We first discuss the space of isoperiodic stable forms for general $g$, and show it is metrically complete. We then explore several aspects of the case $g=2$, which already displays notable complexity. Our analysis of this case provides the first detailed, global picture of the Euclidean geometry of an absolute period leaf, its natural foliation and its discretized Teichmüller dynamics.

Moduli spaces. Let $\mathcal{M}_{g}$ and $\overline{\mathcal{M}}_{g}$ denote the moduli space of compact Riemann surfaces of genus $g$ and its compactification by stable curves. The $g$-dimensional space $\Omega(X)$ of stable forms on $X \in \overline{\mathcal{M}}_{g}$ consists of the holomorphic 1-forms $\omega$ on the smooth locus $X^{*}$ that have at worst simple poles, with opposite residues, at the nodes of $X$. Let $\Omega \overline{\mathcal{M}}_{g}$ denote the moduli space of nonzero stable forms of genus $g$.

Isoperiodic forms. A polarized module is a subgroup $L \subset \mathbb{C}$ isomorphic to $\mathbb{Z}^{2 g}$ equipped with a positive, unimodular symplectic form $[z, w]$. We define the periods of a stable form by

$$
\operatorname{Per}(X, \omega)=\left\{\int_{C} \omega: C \in H_{1}\left(X^{*}, \mathbb{Z}\right)\right\} \subset \mathbb{C} .
$$

Provided it has rank $2 g$, this group is a polarized module with the symplectic form inherited from $H_{1}\left(X^{*}, \mathbb{Z}\right)$.

The moduli space of isoperiodic forms with periods $L$ is defined by

$$
\mathcal{A}(L)=\left\{(X, \omega) \in \Omega \overline{\mathcal{M}}_{g}: \operatorname{Per}(X, \omega)=L \text { as a polarized module }\right\} .
$$

This analytic space of dimension $2 g-3$ will be our main object of study. We begin in $\S 2$ by showing: 
Theorem 1.1 The space of stable forms with fixed absolute periods is isomorphic, as a complex variety, to a standard slice

$$
\mathcal{A}(L)=\overline{\mathcal{S}}_{g} \cap \tilde{H}_{g-1}
$$

of the Schottky locus in Siegel space. Relative period coordinates give $\mathcal{A}(L)$ a stratified complex Euclidean structure, whose underlying path metric is complete.

In particular, we have $\mathcal{A}(L) \cong \mathfrak{H}_{g-1}$ for $g \leq 3$.

The Euclidean coordinates on $\mathcal{A}(L)$ with values in $\mathbb{C}^{s}$ are given by

$$
t_{i}(X, \omega)=\int_{p_{0}}^{p_{i}} \omega
$$

along the stratum where $(X, \omega)$ has exactly $(s+1)$ zeros, $\left(p_{0}, \ldots, p_{s}\right)$. These coordinates describe simple changes in the shapes of polygons on $(X,|\omega|)$, but they are transcendental analytic functions on $\mathfrak{H}_{g}$.

Action of $\mathbf{S L}_{2}(\mathbb{R})$. The space $\Omega \overline{\mathcal{M}}_{g}$ carries a natural action of $\mathrm{SL}_{2}(\mathbb{R})$, satisfying

$$
\operatorname{Per}(\gamma \cdot(X, \omega))=\gamma(\operatorname{Per}(X, \omega)),
$$

where we have identified $\mathbb{C}$ with $\mathbb{R}^{2}$ on the right. The orbits of $\mathrm{SL}_{2}(\mathbb{R})$ project to complex Teichmüller geodesics in $\mathcal{M}_{g}$. The locus $\mathcal{A}(L)$ is stabilized by the subgroup

$$
\mathrm{SL}_{2}(\mathbb{R})^{L}=\{\gamma: \gamma(L)=L \text { and }[\gamma(z), \gamma(w)]=[z, w]\}
$$

which acts on $\mathcal{A}(L)$ by real-linear transformations in period coordinates.

Strata. We let $W(L) \subset \mathcal{A}(L)$ denote the locus where $(X, \omega)$ has a multiple zero, and $P(L)$ the locus where $X$ is a singular curve $\left(X \in \partial \mathcal{M}_{g}\right)$. These loci are invariant under $\mathrm{SL}_{2}(\mathbb{R})^{L}$.

Dynamical classification of forms. It is also useful to classify points in moduli space according to the dynamical properties of the horizontal foliation $\mathcal{F}(\omega)$ of $X$.

We say $\mathcal{F}(\omega)$ is periodic if all its leaves are closed, and we let

$$
\mathcal{A}_{0}(L)=\{(X, \omega) \in \mathcal{A}(L): \mathcal{F}(\omega) \text { is periodic }\} .
$$

This locus is preserved by the action of the upper triangular subgroup $\mathrm{AN}_{2}(\mathbb{R})^{L}$. 
Genus two. In $\S 3$ we begin the study of the case $g=2$. In this case we have $\mathcal{A}(L) \cong \mathbb{H}$ as remarked above.

The Euclidean structure on $\mathcal{A}(L)$ is recorded by the meromorphic quadratic differential $q(L)=d t_{1}^{2}$, which has simple zeros and poles at the points in $W(L)$ and $P(L)$ respectively. The levels sets of $\operatorname{Im}\left(t_{1}\right)$ give a foliation $\mathcal{F}(L)$ of $\mathcal{A}(L)$. Nearby forms on the same leaf of $\mathcal{F}(L)$ are related by sliding their zeros horizontally, while preserving the absolute periods. This operation does not change the dynamics of $\mathcal{F}(\omega)$; in particular, the periodic locus $\mathcal{A}_{0}(L)$ is a union of leaves of $\mathcal{F}(L)$.

The group $\mathrm{SL}_{2}(\mathbb{R})^{L}$ acts on $\mathcal{A}(L)$ by Teichmüller mappings relative to $q(L)$, and its upper triangular subgroup preserves $\mathcal{F}(L)$.

Zero flux. In $\S 4$ we study the case where the periodic locus is nontrivial; that is, we assume

$$
\emptyset \neq \mathcal{A}_{0}(L) \neq \mathcal{A}(L) .
$$

This is equivalent to the zero flux condition:

$$
L \cap \mathbb{R} \text { is a Lagrangian subspace of } L .
$$

(See Proposition 4.1.) In this context we show the Euclidean geometry of $\mathcal{A}_{0}(L)$ can be described as follows.

Theorem 1.2 The periodic locus $\mathcal{A}_{0}(L) \subset \mathbb{H}$ is an open, dense topological disk, tiled by the closures of rectangular strips $R_{i}$ whose upper edges are folded to create leaves of $\mathcal{F}(L)$ with periodic sequences of zeros and poles. All zeros of $q(L)$ are contained in $\mathcal{A}_{0}(L)$.

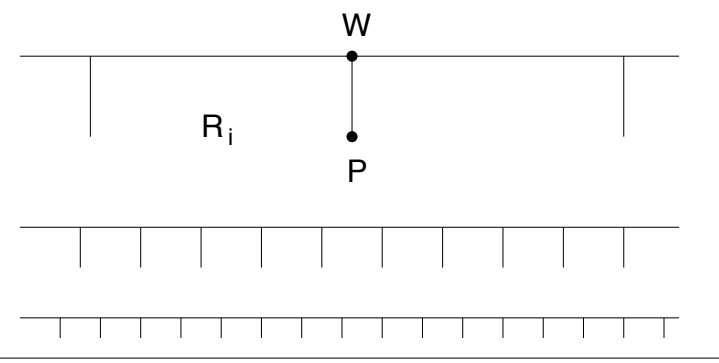

Figure 1 . The region $\mathcal{A}_{0}(L)$ is tiled by rectangular strips, whose edges are folded to create periodic sequences of zeros and poles.

See Figure 1. The interior of each strip is an infinite rectangle, satisfying $\left(R_{i}, q \mid R_{i}\right) \cong\left(\mathbb{R} \times\left(0, h_{i}\right), d z\right)$. The heights $h_{i}$ can be computed from $L$ by 
a continued fraction algorithm. In $\S 4$ we also construct a natural height function

$$
Y: \mathcal{A}_{0}(L) \rightarrow(0, \infty)
$$

whose fibers are the leaves of $\mathcal{F}(L)$, and show:

Theorem 1.3 The tiling of $\mathcal{A}_{0}(L)$ by strips $\bar{R}_{i}$ is mapped by $Y$ to a tiling of $(0, \infty)$ by intervals of length $h_{i}$.

Since $Y$ is bounded below, any unit speed geodesic headed downward in $\left(\mathcal{A}_{0}(L),|q|\right)$ must exit this region in finite time. In particular we easily see:

The region $\mathcal{A}_{0}(L)$ is incomplete in the flat metric $|q|$.

It not at all clear what the limit of such a geodesic should be, and thus completeness of $\mathcal{A}(L)$ is something of a surprise.

Upper degree. To understand how $\mathcal{A}_{0}(L)$ is completed, we turn to the countably many leaves which sweep out the rest of $\mathcal{A}(L)$.

A useful invariant of $\mathcal{F}(\omega)$ is the upper degree $f=\operatorname{deg}^{+}(X, \omega)$, an integer $f \geq 0$ defined in [Mc7] (see $\S 4$ for details). Its level sets give a partition

$$
\mathcal{A}(L)=\bigcup_{f \geq 0} \mathcal{A}_{f}(L)
$$

The induced partition of the poles of $q(L)$ will be denoted by $P(L)=$ $\bigcup_{f \geq 0} P_{f}(L)$.

The upper degree is characterized by the following properties. First, $f=0$ iff $\mathcal{F}(\omega)$ is periodic, so (1.2) is consistent with (1.1). Second, $f$ is constant along the leaves of $\mathcal{F}(L)$. Third, any form in $P_{f}(L)$ can be presented as the connected sum of two forms of genus one joined at a node,

$$
(X, \omega)=(\mathbb{C} / S, d z) \#\left(\mathbb{C} / S^{\perp}, d z\right),
$$

with periods $S$ and $S^{\perp} \subset L$; and we have $f=[\operatorname{Im}(L): \operatorname{Im}(S)]$ provided $f>0$. (Here $\operatorname{Im}(S) \subset \operatorname{Im}(L)$ are the subgroups of $\mathbb{R}$ coming from the imaginary parts of the periods; they are both isomorphic to $\mathbb{Z}^{2}$ when $f>0$.)

Since every leaf of $\mathcal{F}(L)$ outside $\mathcal{A}_{0}(L)$ contains a unique pole, this third property completes the characterization of $f$. The upper degree is also invariant under $\mathrm{AN}_{2}(\mathbb{R})^{L}$.

In $\S 4$ we show: 
Theorem 1.4 The closed set

$$
\partial \mathcal{A}_{0}(L)=\bigcup_{f>0} \mathcal{A}_{f}(L)
$$

is a countable union of leaves of $\mathcal{F}(L)$, each homeomorphic to a ray $[0, \infty)$ based at a pole of $q(L)$.

These leaves produce a complicated comb of repeated folds in the boundary of $\mathcal{A}_{0}(L)$, which renders it complete by Theorem 1.1.

A typical blowup of the foliation $\mathcal{F}(L)$ near $\partial \mathcal{A}_{0}(L)$ is shown in Figure 2. As we will see below, the boundary can be topologically wild and need not be locally connected. It would be interesting to find an explicit model for $\partial \mathcal{A}_{0}(L)$ as a topological space.

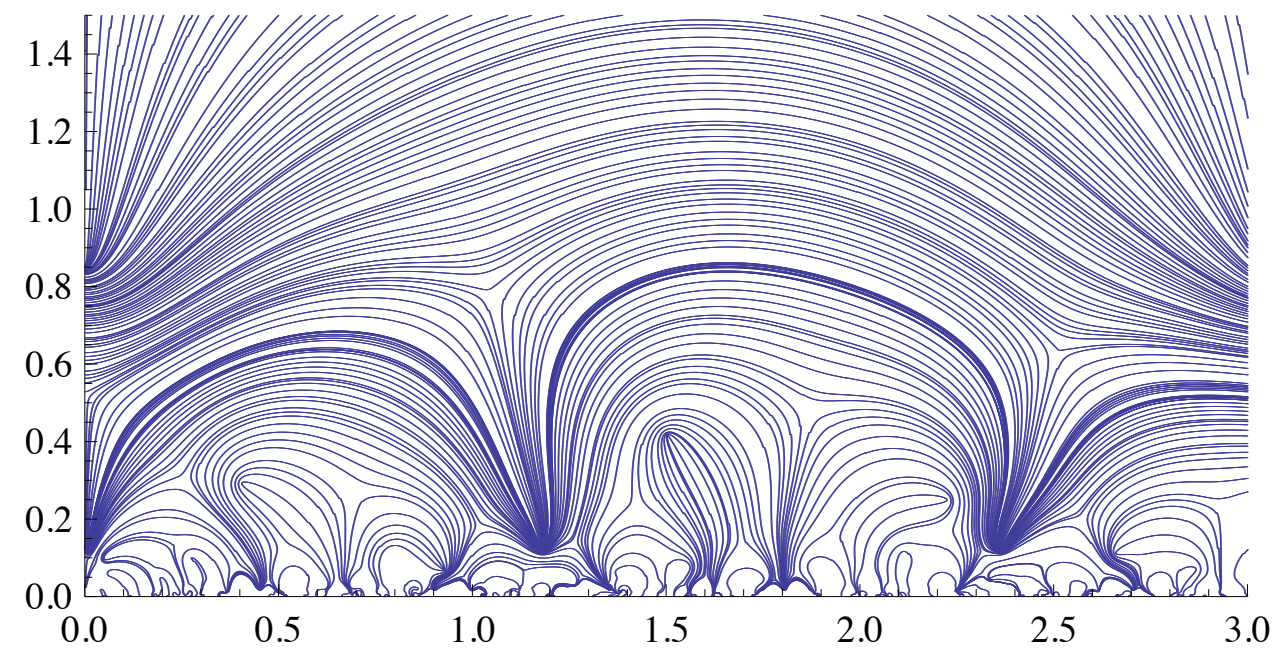

Figure 2. The foliated moduli space $\mathcal{A}\left(L_{D}\right) \cong \mathbb{H}$ near its boundary, for $D=5$.

Cohomological invariants. It is easy to see that the topological type of the foliation $\mathcal{F}(\omega)$ remains constant as $(X, \omega)$ varies along a leaf of $\mathcal{F}(L)$. In particular, any topological invariants of $\mathcal{F}(\omega)$ are constant along leaves.

One such invariant is the content $C(\operatorname{Im} \omega) \subset H^{1}(X, \mathbb{R})$, introduced in [Mc7] (see $\S 4$ ). The content is the smallest convex set containing the cohomology classes $[\operatorname{Im} \omega \mid A]$ for all measurable sets $A$ which are unions of leaves of $\mathcal{F}(\omega)$. In $\S 4$ we also show: 
Theorem 1.5 Two forms in $\mathcal{A}(L)$ determine the same convex set

$$
C(\operatorname{Im} \omega) \subset H^{1}(X, \mathbb{R}) \cong \operatorname{Hom}(L, \mathbb{R})
$$

if and only if they lie on the same leaf of $\mathcal{F}(L)$. Thus the topological type of $\mathcal{F}(\omega)$ is determined by its cohomological invariants.

In particular, periodicity of $\mathcal{F}(\omega)$ is detected by $C(\operatorname{Im} \omega)$.

Eigenforms. In $\S 5$ we turn our attention to isoperiodic forms of genus $g=2$ that satisfy the condition

The locus $\mathrm{SL}_{2}(\mathbb{R}) \cdot \mathcal{A}(L)$ is a closed subset of $\Omega \overline{\mathcal{M}}_{g}$.

This condition insures a certain dynamical completeness for the orbits of $\mathrm{SL}_{2}(\mathbb{R})$ represented in the slice $\mathcal{A}(L)$.

The closure property is equivalent to the condition:

$$
\mathcal{A}(L) \text { consists of eigenforms for real multiplication. }
$$

(See Proposition 5.2.) Thus up to the action of $\mathrm{GL}_{2}^{+}(\mathbb{R})$, the space $\mathcal{A}(L)$ depends only on the discriminant $D>0$ of a real quadratic order

$$
\mathcal{O}_{D} \subset K=\mathbb{Q}(\sqrt{D}) \subset \mathbb{R}
$$

We can therefore reduce to the case where

$$
L_{D}=\mathcal{O}_{D} \oplus \mathcal{O}_{D} i
$$

with the symplectic form

$$
[a+i b, c+i d]=\operatorname{Tr}_{\mathbb{Q}}^{K}\left(D^{-1 / 2}(a d-b c)\right) .
$$

Hilbert modular surfaces. The moduli space $\mathcal{A}\left(L_{D}\right)$ can be identified with a slice $\left\{\tau_{1}\right\} \times \mathbb{H}$ of the universal cover of the Hilbert modular surface

$$
X_{D}=(\mathbb{H} \times \mathbb{H}) / \mathrm{SL}_{2}\left(\mathcal{O}_{D} \oplus \mathcal{O}_{D}^{\vee}\right) .
$$

The normalization (1.3) corresponds to $\tau_{1}=i \sqrt{D}$. Thus $\mathcal{A}\left(L_{D}\right)$ provides an overview of the space of all eigenforms for real multiplication.

The second coordinate on $\mathbb{H} \times \mathbb{H}$ gives a natural isomorphism

$$
\tau_{2}: \mathcal{A}\left(L_{D}\right) \rightarrow \mathbb{H},
$$


with the property that

$$
\operatorname{Jac}(X) \cong \mathbb{C}^{2} / \mathcal{O}_{D} \oplus \mathcal{O}_{D}^{\vee}\left(\tau_{1}, \tau_{2}\right)
$$

for any $(X, \omega) \in \mathcal{A}\left(L_{D}\right)$.

Teichmüller dynamics. The space $\mathcal{A}\left(L_{D}\right)$ also provides a Poincaré section for the action of $\mathrm{SL}_{2}(\mathbb{R})$ on a closed, invariant submanifold of $\Omega \overline{\mathcal{M}}_{g}$. Along $\mathcal{A}\left(L_{D}\right)$, the continuous action of $\mathrm{SL}_{2}(\mathbb{R})$ is replaced the discrete action of the arithmetic group

$$
\mathrm{SL}_{2}(\mathbb{R})^{L_{D}}=\mathrm{SL}_{2}\left(\mathcal{O}_{D}\right) .
$$

The hyperbolic elements in $\mathrm{SL}_{2}\left(\mathcal{O}_{D}\right)$ give examples of the first return map for the Teichmüller geodesic flow. The return time is the same at all points. The action of $\mathrm{SL}_{2}\left(\mathcal{O}_{D}\right)$ on $\mathcal{A}\left(L_{D}\right)$ provides a discretized picture of the dynamics of the Teichmüller geodesic flow, and more generally of the dynamics of $\mathrm{SL}_{2}(\mathbb{R})$ acting on $\Omega \mathcal{M}_{g}$.

Note that $\mathcal{O}_{D}=L_{D} \cap \mathbb{R}$ is Lagrangian, so $\mathcal{A}\left(L_{D}\right)$ enjoys the properties already described in Theorems 1.2 through 1.5 above.

Orbits. By [Mc5], the zeros and poles $W\left(L_{D}\right)$ and $P\left(L_{D}\right)$ of $q\left(L_{D}\right)$ are cut out by the preimages of a pair of algebraic curves $W_{D}$ and $P_{D}$ on $X_{D}$. Since each curve has only finitely many irreducible components, the sets $W\left(L_{D}\right) \cup P\left(L_{D}\right)$ are comprised of finitely many orbits under the action of $\mathrm{SL}_{2}\left(\mathcal{O}_{D}\right)$. In $\S 6$ we also show:

Theorem 1.6 The orbit spaces $W\left(L_{D}\right) / \mathrm{AN}_{2}\left(\mathcal{O}_{D}\right)$ and $P_{f}\left(L_{D}\right) / \mathrm{AN}_{2}\left(\mathcal{O}_{D}\right)$ are finite for each $f \geq 0$.

Since the affine group acts by automorphisms of $\mathcal{F}\left(L_{D}\right)$, this result shows the behavior of a leaf of $\mathcal{F}\left(L_{D}\right)$ is determined up to finitely many choices by its upper degree $f$.

Fixed points and pseudo-Anosov mappings. It is an interesting open problem to determine which elements of $\gamma \in \mathrm{Sp}_{2 g}(\mathbb{Z})$ can be realized by pseudo-Anosov mappings with orientable foliations.

In $\S 7$ we show that, for genus $g=2$, one can usually think of $\gamma$ has a hyperbolic element of $\mathrm{SL}_{2}\left(\mathcal{O}_{D}\right)$ for some $D$. Then $\gamma$ is realizable iff it has a fixed point in $\mathcal{A}\left(L_{D}\right)$. In particular, using the Brouwer fixed point theorem, we show:

Theorem 1.7 Any element $\gamma \in \mathrm{SL}_{2}\left(\mathcal{O}_{D}\right)$ with $\left|\operatorname{Tr}\left(\gamma^{\prime}\right)\right|<2<|\operatorname{Tr}(\gamma)|$ can be realized by an orientable pseudo-Anosov mapping. 
Here $\gamma^{\prime}$ is the Galois conjugate of $\gamma$. Since $\mathcal{O}_{D}$ is dense in $\mathbb{R}$, infinitely many different conjugacy classes can be so realized.

Convergence of leaves. By analyzing the fixed points of elements in $\mathrm{AN}_{2}\left(\mathcal{O}_{D}\right)$, we also establish:

Theorem 1.8 For $f>0$, every path component of $\mathcal{A}_{f}\left(L_{D}\right)$ consists of a leaf of $\mathcal{F}\left(L_{D}\right)$ that starts at a pole $P \in P_{f}\left(L_{D}\right)$ and converges to a point

$$
x \in \mathbb{R}=\partial \mathbb{H}=\partial \mathcal{A}\left(L_{D}\right) .
$$

There is a hyperbolic element $\gamma \in \mathrm{AN}_{2}\left(\mathcal{O}_{D}\right)$ that fixes both $x$ and $P$.

Here we have used the fact that the action of $\gamma$ on $\mathcal{A}\left(L_{D}\right) \cong \mathbb{H}$ is quasiconformal, so it extends to a homeomorphism on $\partial \mathbb{H}$. (In fact, $\gamma \in \mathrm{SL}_{2}\left(\mathcal{O}_{D}\right)$ acts on $\partial \mathbb{H}$ via the Möbius transformations $\gamma^{\prime}(x)$ defined by its Galois conjugate - see (6.1) below).

Inaccessible points. Recall that a point $x \in \partial U$ is accessible if there is an arc $\alpha \subset \bar{U}$ meeting $\partial U$ only at $x$. Using the dynamics of the affine group, in $\S 7$ we also show:

Theorem 1.9 The boundary points of $\mathcal{A}_{0}(L)$ that lie in $\mathcal{A}_{f}\left(L_{D}\right)$ are inaccessible for all $f \gg 0$. In particular, $\partial \mathcal{A}_{0}\left(L_{D}\right)$ is not locally connected.

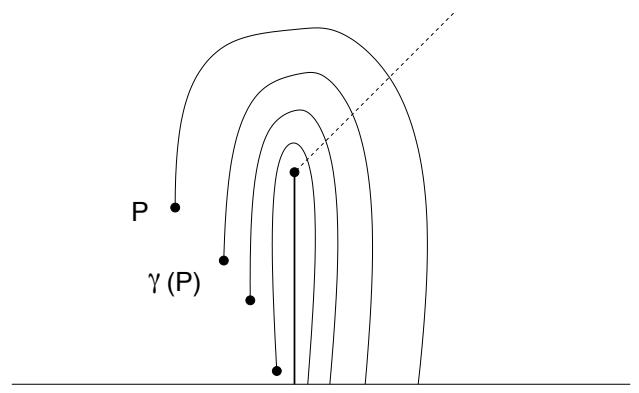

Figure 3. Inaccessible points in $\partial \mathcal{A}_{0}\left(L_{D}\right)$.

The mechanism that creates inaccessible leaves is shown in Figure 3. Suppose a pole $Q$ of $q\left(L_{D}\right)$ in $\partial \mathcal{A}_{0}\left(L_{D}\right)$ is stabilized by a hyperbolic element $\gamma \in \mathrm{AN}_{2}\left(\mathcal{O}_{D}\right)$. If the stable manifold for $\gamma$ (shown as a dotted line) crosses the leaf through another pole $P \in \partial \mathcal{A}_{0}\left(L_{D}\right)$, then by iterating $\gamma$ we obtain a sequence of leaves that make $Q$ inaccessible from within the periodic locus. 

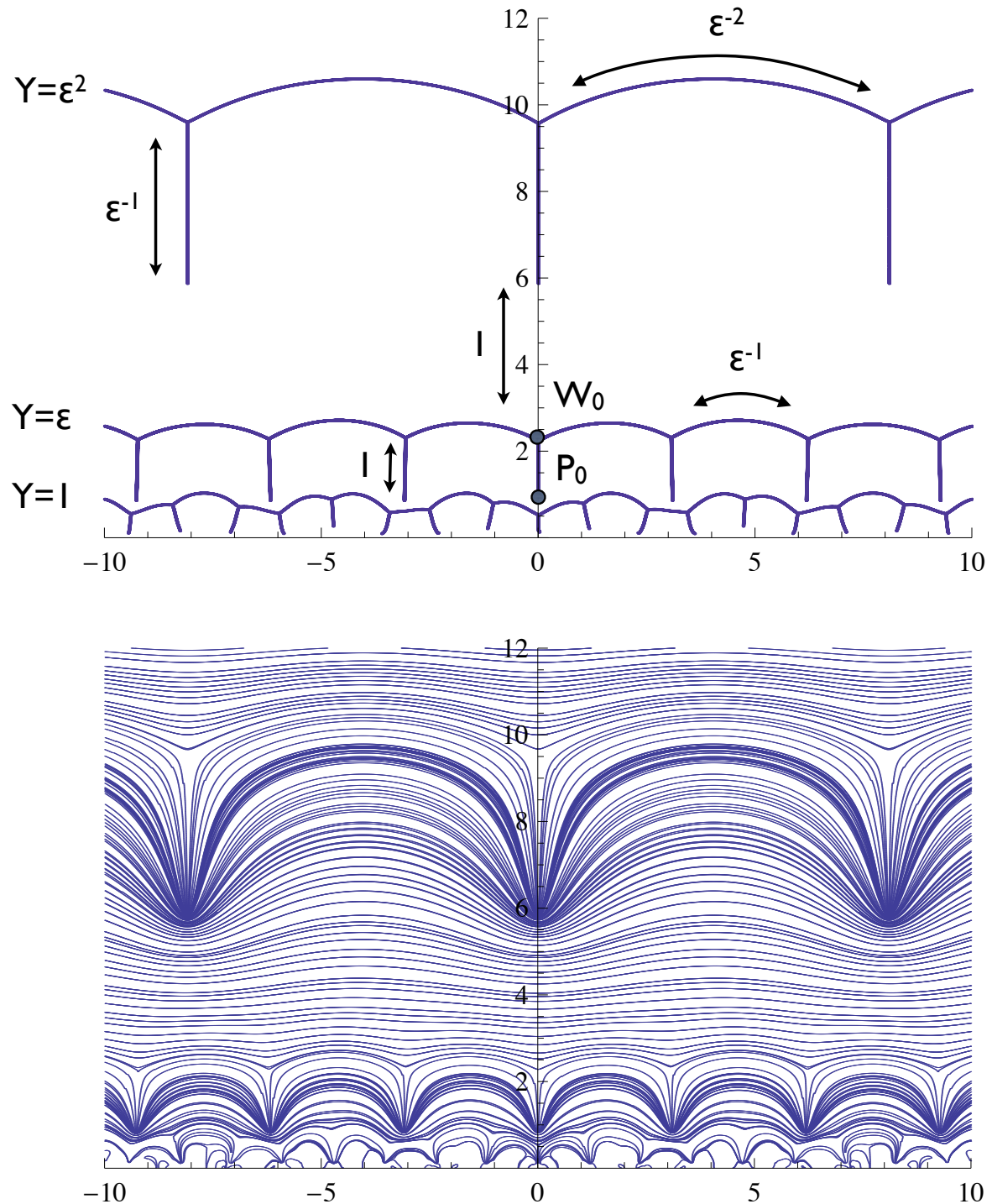

Figure 4. Top: Two adjacent strips in $\mathcal{A}\left(L_{D}\right)$ for $D=5$. Indicated lengths are measured in the $\left|q\left(L_{D}\right)\right|$ metric.

Bottom: The same region, with the foliation $\mathcal{F}\left(L_{D}\right)$. 
The case $\boldsymbol{D}=\mathbf{5}$. Next we provide a more detailed description of $\mathcal{A}(D)$ in the case $D=5$. Let $\epsilon=(1+\sqrt{5}) / 2$ be the fundamental unit of $\mathcal{O}_{D}$.

As we will see in $\S 8$, there is a distinguished zero $W_{0}$ of $q\left(L_{D}\right)$ with coordinate $\tau_{2}\left(W_{0}\right)=i \sqrt{D}$ which represents an eigenform for complex multiplication. The remaining zeros $W_{n, k}$ of $q\left(L_{D}\right)$ form a single orbit under $\mathrm{AN}_{2}\left(\mathcal{O}_{D}\right)$ which can be effectively enumerated with two indices $(n, k)$. We have $Y\left(W_{n, k}\right)=\epsilon^{n}$, and these level sets define the boundaries of the strips making up $\mathcal{A}_{0}\left(L_{D}\right)$. Each zero is connected by a leaf of $\mathcal{F}\left(L_{D}\right)$ to a unique pole $P_{n, k}$ which satisfies

$$
\tau_{2}\left(P_{n, k}\right)=\epsilon^{4 n-2} \tau_{1}+5 k \epsilon^{2 n-1},
$$

where $\tau_{1}=i \sqrt{D}$.

This geometry is illustrated in Figure 4(top). The full foliation $\mathcal{F}\left(L_{D}\right)$ in the same region is shown in Figure 4, and a blowup near the real axis is given in Figure 2.

Fake pentagons, class numbers and cascades. We conclude by briefly elaborating three other topics in the case $D=5$.

In $\S 9$ we use the enumeration of $W\left(L_{D}\right)$ to effectively describe all fake pentagons. The latter are forms of genus two with double zeros, such that $\operatorname{Per}(X, \omega) \cong \mathbb{Z}\left[\zeta_{5}\right]$ as a polarized module. One such form comes from the regular pentagon, but there are countably many others (compare [Mc5, §9]).

In $\S 10$ we give an example of the relationship to number theory, by showing:

Theorem 1.10 For $D=5$ and $f>0$, the number of orbits of $\mathrm{AN}_{2}\left(\mathcal{O}_{D}\right)$ in $P_{f}\left(L_{D}\right)$ is given by the strict class number $h^{+}\left(f^{2} D\right)$.

Finally in $\S 11$ we give an explicit family of forms $\left(X_{t}, \omega_{t}\right)$ of genus 2 such that foliations $\mathcal{F}\left(i \omega_{t}\right)$ bifurcate for a countable set of $t$ homeomorphic to $\omega^{\omega}$. These bifurcation give some indication of the way in which the countably many leaves in $\bigcup_{f>0} \mathcal{A}_{f}\left(L_{D}\right)$ intervene when a Euclidean geodesic that starts in $\mathcal{A}_{0}\left(L_{D}\right)$ reaches its boundary; instead of leaving the space, it is forced to double back into it.

Higher-dimensional examples. When $g=3$, we have $\mathcal{A}(L) \cong \mathfrak{H}_{2}$. In this case one can mimic the construction of $L_{D}$ to show:

Proposition 1.11 For any totally real cubic field $K$, there is a natural action of $\mathrm{SL}_{2}\left(\mathcal{O}_{K} \oplus \mathcal{O}_{K}^{\vee}\right)$ on the three-dimensional Siegel space $\mathfrak{H}_{2}$. 
In contrast to the case of quadratic fields, most points in $\mathfrak{H}_{2}$ do not represent eigenforms. It would be interesting to investigate these genus three examples in more detail.

For genus $g \geq 4$, the study of $\mathcal{A}(L)$ implicitly involves the Schottky problem, as can be seen from Theorem 1.1. It appears to be unknown if $\mathcal{A}(L)$ is connected for $g \geq 4$. For related connectedness results, see [KZ] and [La].

Notes and references. Figures 2, 4 and 12 were created using an elegant theta function formula for $q\left(L_{D}\right)$ due to Möller and Zagier; see $\S 5$.

This paper is a sequel to [Mc5] and [Mc7], and continues an investigation begun in [Mc1]. The proof of completeness of $\mathcal{A}(L)$ (Theorem 1.1) relies on [Mc6], which provides additional background. The geometry of the curves $W_{D}$ and $P_{D}$ on the Hilbert modular surface $X_{D}$ is developed in [Ba]; see also [Mu]. Related work on genus 2 covers of elliptic curves appears in $[\mathrm{Sch}]$. Some results related to transverse invariant measures for $\mathcal{F}(L)$ appear in $[\mathrm{CW}]$. For general background on $\Omega \mathcal{M}_{g}$ and related topics in Teichmüller theory, see e.g. the surveys [Z] and [Mo]. The variety $\mathcal{A}(L)$ can also be regarded as the space of isomonodromic deformations of the differential equation $y^{\prime}+\omega y=0$; for more on this broader setting, see e.g. [Bol]

I would like to thank R. Mukamel for useful conversations related to this work.

\section{Slices of Schottky space}

In this section we describe $\mathcal{A}(L)$ for general genus $g$ and establish Theorem 1.1. More generally, we discuss the moduli space of holomorphic 1-forms representing a fixed cohomology class $\phi \in H^{1}\left(\Sigma_{g}, \mathbb{C}\right)$.

Compact type. A stable curve $X \in \overline{\mathcal{M}}_{g}$ is of compact type if the following equivalent conditions are satisfied:

1. The Jacobian of $X$ is compact.

2. The stable forms $\omega \in \Omega(X)$ have no poles.

3. The irreducible components of $X$ form a tree.

4. Their genera satisfy $\sum g_{i}=g$.

Schottky space. Let $\Sigma_{g}$ be an oriented topological surface of genus $g$. Let $\langle\alpha, \beta\rangle=(i / 2) \int_{\Sigma_{g}} \alpha \wedge \bar{\beta}$. denote the intersection form on $H^{1}(\Sigma, \mathbb{C})$. 
The universal cover of $\mathcal{M}_{g}$ can be identified with the Teichmüller space $\mathcal{T}_{g}$ of Riemann surfaces marked by $\Sigma_{g}$. Its quotient by the Torelli group gives the Schottky space $\mathcal{S}_{g}$.

A point in $\mathcal{S}_{g}$ is specified by a pair $[X, m]$ consisting of a Riemann surface plus a symplectic isomorphism or marking

$$
m: H^{1}\left(\Sigma_{g}, \mathbb{C}\right) \cong H^{1}(X, \mathbb{C})
$$

that sends $H^{1}\left(\Sigma_{g}, \mathbb{Z}\right)$ to $H^{1}(X, \mathbb{Z})$. A useful completion $\overline{\mathcal{S}}_{g}$ is obtained by allowing $X$ to be a stable curve of compact type. By taking the period matrix of such $X$, we obtain an analytic inclusion

$$
\overline{\mathcal{S}}_{g} \subset \mathfrak{H}_{g}
$$

whose image is the Schottky locus, the closed analytic subvariety of Siegel space coming from the Jacobians of stable curves of compact type. As is well-known, we have $\overline{\mathcal{S}}_{g}=\mathfrak{H}_{g}$ iff $g \leq 3$.

Absolute period leaves. The space $\Omega \mathcal{M}_{g}$ carries a natural absolute period foliation $\mathcal{A}$, with the property that $[\omega] \in H^{1}(X, \mathbb{C})$ is locally constant along its leaves. This condition defines an analytic foliation transverse to the orbits of $\mathrm{SL}_{2}(\mathbb{R}$ ), with leaves of dimension $2 g-3$ (for $g \geq 2$ ). Each leaf carries a stratified geometric structure coming from the relative periods, which are determined by integrating $\omega$ along paths joining its zeros. (See e.g. [Mc6, $\S 3]$.)

Period slices. Now fix a positive cohomology class $\phi \in H^{1}(X, \mathbb{C})$ (meaning $\langle\phi, \phi\rangle>0)$. Let

$$
\mathcal{S}_{g}(\phi)=\left\{[X, m]: m(\phi) \in H^{1,0}(X)\right\},
$$

and let $\pi([X, m])=(X, \omega) \in \Omega \mathcal{M}_{g}$, where $[\omega]=\phi$. The definitions readily imply:

Proposition 2.1 Each leaf of the absolute period foliation is swept out by a component of $\mathcal{S}_{g}(\phi)$ under the projection map

$$
\pi: \mathcal{S}_{g}(\phi) \rightarrow \Omega \mathcal{M}_{g}
$$

\section{Characterization of holomorphic forms. Let}

$$
\operatorname{Per}(\phi)=\left\{\phi(C): C \in H_{1}\left(\Sigma_{g}, \mathbb{Z}\right)\right\} \subset \mathbb{C} .
$$

We say $\phi$ is an elliptic cohomology class, of degree $d>0$, if $\operatorname{Per}(\phi) \cong \mathbb{Z}^{2}$ is a lattice in $\mathbb{C}$ and if the natural map $\Sigma_{g} \rightarrow \mathbb{C} / \operatorname{Per}(\phi)$ has degree $d$. (Since $\phi$ is positive, so is $d$. .) By [Kap] we have: 
Proposition 2.2 The slice $\mathcal{S}_{g}(\phi)$ is nonempty unless $\phi$ is elliptic of degree one.

Completions. By allowing $X$ to be a stable curve of compact type, we can similarly define

$$
\pi: \overline{\mathcal{S}}_{g}(\phi) \rightarrow \Omega \overline{\mathcal{M}}_{g}
$$

The advantage here is that the completion is simply a linear slice of the Schottky locus.

Proposition 2.3 We have $\overline{\mathcal{S}}_{g}(\phi)=\overline{\mathcal{S}}_{g} \cap \mathfrak{H}_{g-1}$ for some standard copy of $\mathfrak{H}_{g-1}$ inside of $\mathfrak{H}_{g}$.

Proof. Choose a real symplectic basis $\left(a_{i}, b_{i}\right)$ of $H_{1}\left(\Sigma_{g}, \mathbb{R}\right)$ such that $\phi\left(a_{1}\right)=1$ and $\phi\left(a_{i}\right)=\phi\left(b_{i}\right)=0$ for $i>1$. Then $\phi\left(b_{1}\right)=\sigma \in \mathbb{H}$. For any marked Abelian variety $(J, m)$, the unique forms $\omega_{i} \in \Omega(J)$ such that $\omega_{i}\left(a_{j}\right)=\delta_{i j}$ determine the associated period matrix $\tau_{i j}=\omega_{i}\left(b_{j}\right) \in \mathfrak{H}_{g}$.

Clearly $\phi \in H^{1,0}(J)$ iff $\phi=\left[\omega_{1}\right]$, which is equivalent to the condition that $\tau_{11}=\sigma$ and $\tau_{1 i}=0$ for $i>1$. This means simply that $\tau=[\sigma] \oplus \tau^{\prime}$ lies in a standard copy of $\mathfrak{H}_{g-1}$ inside $\mathfrak{H}_{g}$. By intersecting with the Schottky locus, we insure that $J$ is a Jacobian.

Proposition 2.4 The locus $\overline{\mathcal{S}}_{g}(\phi)$ is nonempty for every positive class $\phi$.

Proof. A stable form realizing $\phi$ on a stable curve with components of genus 1 and $g-1$ is easily constructed when $\phi$ is elliptic of degree one, and the other cases are handled by Proposition 2.2.

Analytic symmetries. Fix a symplectic basis for $H_{1}\left(\Sigma_{g}, \mathbb{Z}\right)$. We may then identify $\mathrm{Sp}_{2 g}(\mathbb{R})$ and $\mathrm{Sp}_{2 g}(\mathbb{Z})$ with the groups of automorphisms of $H^{1}(X, \mathbb{C})$ that preserve $H^{1}(X, \mathbb{R})$ and $H^{1}(X, \mathbb{Z})$ respectively. The action of a symplectic transformation $\gamma$ on a cohomology class $\alpha$ will be denoted by $\gamma \cdot \alpha$.

Let $\operatorname{Sp}_{2 g}(\mathbb{R})^{\Phi}$ denote the subgroup of $\operatorname{Sp}_{2 g}(\mathbb{R})$ stabilizing the real 2dimensional subspace

$$
\Phi=\langle\operatorname{Re}(\phi), \operatorname{Im}(\phi)\rangle .
$$

Then the symplectic complement $\Phi^{\perp} \cong \mathbb{R}^{2 g-2}$ is also preserved, and we have

$$
\operatorname{Sp}_{2 g}(\mathbb{R})^{\Phi} \cong \mathrm{SL}_{2}(\mathbb{R}) \times \operatorname{Sp}_{2 g-2}(\mathbb{R})
$$


The projection of a symplectic automorphism $\gamma$ to the first factor is given by the matrix $D \gamma \in \mathrm{SL}_{2}(\mathbb{R})$ satisfying

$$
(\gamma \cdot \phi)=D \gamma \circ \phi: H_{1}\left(\Sigma_{g}, \mathbb{Z}\right) \rightarrow \mathbb{C} \cong \mathbb{R}^{2} .
$$

The kernel of $D$ is the same as the subgroup $\operatorname{Sp}_{2 g}(\mathbb{R})^{\phi}$ stabilizing $\phi$. Since the integral points this group simply change the marking, we have:

Proposition 2.5 The fibers of $\pi: \overline{\mathcal{S}}_{g}(\phi) \rightarrow \Omega \overline{\mathcal{M}}_{g}$ are the orbits of $\operatorname{Sp}_{2 g}(\mathbb{Z})^{\phi}$.

Remark: the absolute period foliation in low genus. When $g \leq 3$, the absolute period foliation $\mathcal{A}$ of $\Omega \mathcal{M}_{g}$ can be understood using results on group actions on homogeneous spaces. For example, letting $\Omega_{1} \mathcal{M}_{g}$ denote the locus where $\int_{X}|\omega|^{2}=1$, we have:

Proposition 2.6 Provided $g \leq 3$, the foliation $\mathcal{A}$ of $\Omega_{1} \mathcal{M}_{g}$ is ergodic and its closed leaves all come from elliptic cohomology classes.

Proof. Since $\overline{\mathcal{S}}_{g}=\mathfrak{H}_{g}$ for $g \leq 3$, Proposition 2.3 shows the leaves of $\mathcal{A}$ are covered by totally geodesic submanifolds $\mathfrak{H}_{g-1} \subset \mathfrak{H}_{g}$. Thus the study of $\mathcal{A}$ can be reduced to the study of the action of $\mathrm{Sp}_{2 g-2}(\mathbb{R})$ on $\mathrm{Sp}_{2 g}(\mathbb{R}) / \mathrm{Sp}_{2 g}(\mathbb{Z})$. This action is ergodic by Moore's theorem, so $\mathcal{A}$ is ergodic as well. Similarly, by Ratner's theorems [Rat], the leaf of $\mathcal{A}$ defined by $\phi: H_{1}\left(\Sigma_{g}, \mathbb{R}\right) \rightarrow \mathbb{C}$ is closed iff

$$
\Gamma=G \cap \mathrm{Sp}_{2 g}(\mathbb{Z})
$$

is a lattice in $G=\mathrm{Sp}_{2 g}(\mathbb{R})^{\phi} \cong \operatorname{Sp}_{2 g-2}(\mathbb{R})$.

Note that $G$ acts faithfully on the symplectic space $\operatorname{Ker}(\phi) \subset H_{1}\left(\Sigma_{g}, \mathbb{R}\right)$ of rank $2 g-2$, and $G$ acts by the identity on $\operatorname{Ker}(\phi)^{\perp} \cong \mathbb{R}^{2}$. The subgroup $\Gamma$ preserves the integral points $H_{1}\left(\Sigma_{g}, \mathbb{Z}\right)$, so it also preserves the subgroup $L=\operatorname{Ker}(\phi) \cap H_{1}\left(\Sigma_{g}, \mathbb{Z}\right)$. We have $L \cong \mathbb{Z}^{s}$ with $0 \leq s \leq 2 g-2$. If $s=0$ then $H_{1}\left(\Sigma_{g}, \mathbb{Z}\right)$ projects faithfully to $\operatorname{Ker}(\phi)^{\perp}$; since $G$ acts trivially there, $\Gamma$ is also trivial. If $0<s<2 g-2$, then $\Gamma$ preserves the subspaces $\mathbb{R} \cdot L \subset \operatorname{Ker}(\phi)$, so it is not Zariski dense in $G$. Thus $\Gamma$ can only be a lattice when $s=2 g-2$. But this is exactly the case where $\phi$ is elliptic.

Conversely, it is readily verified that an elliptic class $\phi$ of degree $d$ defines a closed leaf of $\mathcal{A}$, corresponding to degree $d$ covers of the fixed elliptic curve $\mathbb{C} / \operatorname{Per}(\phi)$. 
It would be interesting to have a similar analysis for $g \geq 4$.

Quasiconformal symmetries. The holomorphic action of $\operatorname{Sp}_{2 g}(\mathbb{Z})^{\phi}$ extends to a quasiconformal action of $\operatorname{Sp}_{2 g}(\mathbb{Z})^{\Phi}$ on $\overline{\mathcal{S}}_{g}(\phi)$, defined as follows.

Suppose $\gamma \in \operatorname{Sp}_{2 g}(\mathbb{Z})^{\Phi}$ and $[X, m] \in \overline{\mathcal{S}}_{g}(\phi)$. Let $A=D \gamma$ and let $\left(X_{A}, \omega_{A}\right)=A \cdot(X, \omega)$, where $\pi([X, m])=(X, \omega)$. Then the natural homeomorphism $X \cong X_{A}$ gives a marking $m_{A}$ of $X_{A}$ sending $A \circ \phi$ to $\left[\omega_{A}\right]$. But $A \circ \phi=\gamma \cdot \phi$, so

$$
f_{\gamma}([X, m])=\left(X_{A}, m_{A} \circ \gamma\right)
$$

lies in $\overline{\mathcal{S}}_{g}(\phi)$. The action is defined by $[X, m] \mapsto f_{\gamma}([X, m])$.

Foliation picture. Alternatively, one can observe that the standard analytic action of $\operatorname{Sp}_{2 g}(\mathbb{Z})^{\Phi}$ on $\mathfrak{H}_{g}$ preserves the locus of marked Abelian varieties

$$
\mathfrak{H}_{g}(\Phi)=\left\{[J, m]: \Phi=\langle\operatorname{Re} \omega, \operatorname{Im} \omega\rangle \text { for some } \omega \in H^{1,0}(J)\right\}
$$

which is isomorphic to $\mathbb{H} \times \mathfrak{H}_{g-1}$. The Schottky sublocus $\overline{\mathcal{S}}_{g}(\Phi)=\overline{\mathcal{S}}_{g} \cap \mathfrak{H}_{g}(\Phi)$ is preserved as well, and foliated by the Teichmüller disks generated by $\mu=\bar{\omega} / \omega$. Each of these disks has a unique intersection with the transversal $\overline{\mathcal{S}}_{g}(\phi)$, and so the analytic action of $\operatorname{Sp}_{2 g}(\mathbb{Z})^{\Phi}$ on the space of leaves gives a topological action on this transversal.

From this perspective we immediately see:

Proposition 2.7 Two points of $\overline{\mathcal{S}}_{g}(\phi)$ lie in the same orbit of $\mathrm{Sp}_{2 g}(\mathbb{Z})^{\Phi}$ iff they project into the same orbit of $\mathrm{SL}_{2}(\mathbb{R})$ on $\Omega \overline{\mathcal{M}}_{g}$.

Period coordinates and stratified Euclidean structure. The space $\mathcal{S}_{g}(\phi)$ breaks into strata along which $|Z(\omega)|$, the number of zeros of the form with $[\omega]=m(\phi)$, is locally constant. Let $G=S_{s}$ be the symmetric group, acting on $\mathbb{X}=\mathbb{C}^{s} /(1,1, \ldots, 1)$ by permuting coordinates. If we label the zeros of $\omega$ as $\left(p_{1}, \ldots, p_{s}\right)$, and choose a basepoint $q$, then the relative periods $t_{i}=\int_{q}^{p_{i}} \omega$ provide each stratum with the structure of a $(G, \mathbb{X})$ manifold (see [Mc6, §3]). The action of $f_{\gamma}$ on $\operatorname{Sp}_{2 g}(\mathbb{Z})^{\Phi}$ on $\mathcal{S}_{g}(\phi)$ is also linearized in these coordinates, with derivative $D f_{\gamma}=(D \gamma, \ldots, D \gamma) \in\left(\mathrm{SL}_{2}(\mathbb{R})\right)^{s}$ on $\mathbb{C}^{s}$.

Full support. The relative period charts just described extend to $\overline{\mathcal{S}}_{g}(\phi)$, provided we exclude those forms $(X, \omega)$ which vanish identically on some irreducible component of $X$. (In the case of a stable curve, the nodes of $X$ should be included in the list $\left(p_{1}, \ldots, p_{s}\right)$.)

Such a component would give a pair $a, b \in H_{1}\left(\Sigma_{g}, \mathbb{Z}\right)$ with $\phi(a)=\phi(b)=$ 0 and $a \cdot b=1$. If no such pair exists, we say $\phi$ has full support. We then have: 
Proposition 2.8 If $\phi$ has full support, then $\operatorname{Sp}_{2 g}(\mathbb{Z})^{\Phi}$ acts on $\overline{\mathcal{S}}_{g}(\phi)$ by real linear transformations in stratified relative period coordinates.

Note: if $\phi$ does not have full support, then we construct a stable form $(X, \omega)$ in $\pi\left(\overline{\mathcal{S}}_{g}(\phi)\right)$ such that one irreducible component $X_{0}$ of $X$ is an elliptic curve, $H_{1}\left(X_{0}, \mathbb{Z}\right)=\langle a, b\rangle$, and $\omega \mid X_{0}=0$.

Completeness. In the case of full support, each stratum carries a natural path metric coming from the norm $\left\|\left(z_{i}\right)\right\|=\max \left|z_{i}-z_{j}\right|$ on $\mathbb{C}^{s} /(1,1, \ldots, 1)$ on relative periods.

Proposition 2.9 The space $\overline{\mathcal{S}}_{g}(\phi)$ is complete in the relative period metric provided $\operatorname{Per}(\phi) \cong \mathbb{Z}^{2 g}$.

Proof. Let $p:[0,1) \rightarrow \overline{\mathcal{S}}_{g}(\phi)$ be a path of finite length in the relative period metric. By [Mc6, Theorem 1.4], the path $\left(X_{t}, \omega_{t}\right)=\pi \circ p(t)$ has a limit $(X, \omega) \in \Omega \overline{\mathcal{M}}_{g}$ provided we ignore the components of the limit on which $\omega$ vanishes identically. But $\phi$ has full support, so there are no such components; and hence $[\omega]=\phi$ for a suitable marking of $X$. Finally $\operatorname{Jac}(X)$ is compact, since $\phi\left(H_{1}\left(\Sigma_{g}, \mathbb{Z}\right)\right) \cong \mathbb{Z}^{2 g}$.

Proof of Theorem 1.1. Choose a symplectic isomorphism $\phi: H_{1}\left(\Sigma_{g}, \mathbb{Z}\right) \rightarrow$ $L$. Then we may identify $\mathcal{A}(L)$ with $\overline{\mathcal{S}}_{g}(\phi)$ and apply the results above.

Examples of incompleteness. If $\operatorname{Per}(\phi)$ is not isomorphic to $\mathbb{Z}^{2 g}$, then $\overline{\mathcal{S}}_{g}(\phi)$ is incomplete. Indeed, whenever $C \in H_{1}\left(\Sigma_{g}, \mathbb{Z}\right)$ is a primitive class in the kernel of $\phi$, we can find a path of finite length in $\overline{\mathcal{S}}_{g}(\phi)$ whose image in $\Omega \overline{\mathcal{M}}_{g}$ converges to a stable form $(X, \omega)$ such that $C$ is pinched to become a node. This path has no limit in $\overline{\mathcal{S}}_{g}(\phi)$, since $\operatorname{Jac}(X)$ is not compact.

\section{Genus two}

This section introduces charts and notation for the study of $\mathcal{A}(L)$ in the case $g=2$.

Polarized modules. We define the determinant of a polarized module $L \subset \mathbb{C}$ by

$$
\operatorname{det}(L)=\sum_{i=1}^{g} \operatorname{Im}\left(\bar{a}_{i} b_{i}\right),
$$

where $\left(a_{i}, b_{i}\right)$ is an symplectic basis for $L$ and $\operatorname{Im}(\bar{z} w)$ is the usual symplectic form on $\mathbb{C}$. We say $L$ is positive if $\operatorname{det}(L)>0$. 
Any polarized module $L \subset \mathbb{C}$ can be regarded as the image of a symplectic isomorphism $\phi: H_{1}\left(\Sigma_{g}, \mathbb{Z}\right) \rightarrow L$ with $\langle\phi, \phi\rangle=\operatorname{det}(L)$. The choice of $\phi$ determines a bijection

$$
\overline{\mathcal{S}}_{g}(\phi) \cong \mathcal{A}(L)
$$

together with a compatible isomorphism

$$
\mathrm{Sp}_{2 g}(\mathbb{Z})^{\Phi} \cong \mathrm{SL}_{2}(\mathbb{R})^{L}
$$

Thus $\mathcal{A}(L)$ is a special case of the moduli space $\overline{\mathcal{S}}_{g}(\phi)$. In terms of the standard action of $\mathrm{SL}_{2}(\mathbb{R})$ on $\Omega \mathcal{M}_{g}$, we have (using 3.1)

$$
f_{\gamma}(X, \omega)=\gamma \cdot(X, \omega)
$$

for all $\gamma \in \mathrm{SL}_{2}(\mathbb{R})^{L}$.

Genus two. Now suppose $g=2$. Then we have $\mathcal{A}(L) \cong \overline{\mathcal{S}}_{g}(\phi)=\mathbb{H}$. The unique relative period

$$
t=\int_{p_{1}}^{p_{2}} \omega
$$

gives rise to a globally defined meromorphic quadratic differential $q(L)=d t^{2}$ on $\mathcal{A}(L)$. As in $[\mathrm{Mc} 5, \S 8]$, a local calculation shows that the zeros and poles of $q(L)$ are simple, and coincide with the sets $W(L)$ and $P(L)$ respectively.

Foliations. We let $\mathcal{F}(L)$ denote the horizontal foliation of $\mathcal{A}(L)$ determined by the quadratic differential $q(L)$. The leaves of $\mathcal{F}(L)$ are horizontal lines in the relative period coordinate $t$.

Dynamics. For each $\gamma \in \mathrm{SL}_{2}(\mathbb{R})^{L}$ the derivative of $f_{\gamma}$ in local coordinates where $q=(d x+i d y)^{2}$ is given by

$$
D_{q} f_{\gamma}=\gamma
$$

This is simply because the relative periods of $\gamma \cdot(X, \omega)$ are the image of the relative periods of $(X, \omega)$ under $\gamma$.

When $\gamma \in \mathrm{AN}_{2}(\mathbb{R})^{L}$, the map $f_{\gamma}$ it also preserves the foliation $\mathcal{F}(L)$. These two properties often assist in explicitly determining the action of $f_{\gamma}$, especially once a fixed point is known.

Splittings. Any submodule $S=\mathbb{Z} a \oplus \mathbb{Z} b \subset L$ with $[a, b]=1$ determines a symplectic splitting

$$
L=S \oplus S^{\perp} .
$$

The splitting is positive if $\operatorname{det}(S)>0$ and $\operatorname{det}\left(S^{\perp}\right)>0$; equivalently, if $S$ and $S^{\perp}$ are positively-oriented rank two lattices in $\mathbb{C}$. 
Charts. We wish to describe some complex charts where $q(L)=d t^{2}$. Given a positive splitting $L=S \oplus S^{\perp}$, let $U_{S} \subset \mathbb{C}$ be the open region obtained by removing the rays $[1, \infty) \cdot \lambda$ from $\mathbb{C}$ for every $\lambda \neq 0$ in $S \cup S^{\perp}$.

Using connected sums [Mc4, §7], for $t \in U_{S}$ we define

$$
X_{S}(t)=(\mathbb{C} / S, d z) \underset{[0, t]}{\#}\left(\mathbb{C} / S^{\perp}, d z\right) .
$$

Here the 1-form $\left(X_{t}, \omega_{t}\right)=X_{S}(t)$ is constructed by cutting open the flat tori on the right and the left along the projection of $[0, t]$, and then gluing the resulting slits together. For $t=0$ we obtain a stable form on a pair of elliptic curves joined at node; otherwise we obtain a form with two distinct zeros on a smooth Riemann surface of genus two.

Proposition 3.1 Any positive splitting $L=S \oplus S^{\perp}$ determines a holomorphic map

$$
X_{S}: U_{S} \rightarrow \mathcal{A}(L)
$$

such that $X_{S}(t)=X_{S}(-t)$ and $X_{S}^{*}(q(L))=d t^{2}$. This map is an immersion for $t \neq 0$.

Proof. The absolute periods of $(X, \omega)=X_{S}(t)$ are given by $S \oplus S^{\perp}=L$, and by construction the relative period is $t$.

The map $t \mapsto X_{S}(t)$ is a branched covering of degree two at $t=0$. Because of this branching, $q(L)$ has a simple pole at $X_{S}(0)$. The map $U_{S} /( \pm 1) \rightarrow \mathcal{A}(L)$ is a local homeomorphism.

Points of $\boldsymbol{P}(\boldsymbol{L})$ and $\boldsymbol{W}(\boldsymbol{L})$. Clearly a positive splitting gives a stable form $X_{S}(0) \in P(L)$, and every form in $P(L)$ arises in this way. Indeed, since $\operatorname{Jac}(X)$ is compact, any $(X, \omega) \in P(L)$ gives a pair of elliptic curves whose periods give a splitting of $L$.

Every form of genus two with distinct zeros can be presented as a connected sum $[\mathrm{Mc} 4, \S 7]$, so the charts $X_{S}\left(U_{S}\right)$ cover $\mathcal{A}(L)-W(L)$. The connected sum extends to $t \neq 0$ in $S$ provided $(0, t) \cap\left(S \cup S^{\perp}\right)=\emptyset$. The result is a form with a double zero $X_{S}(t) \in W(L)$; and again, all points of $W(L)$ arise in this way.

\section{Zero flux}

In this section we continue the analysis of genus two, under the added assumption: 
$L \cap \mathbb{R}$ is a Lagrangian subspace of $L$.

We give a concrete picture of the periodic locus $\mathcal{A}_{0}(L)$, and establish Theorems 1.2 through 1.5 .

Zero flux. We begin by recapitulating some results from [Mc7].

Let $\rho$ be a smooth closed 1-form on a closed, oriented $n$-manifold $M$, with periods $P=\operatorname{Per}(\rho) \subset \mathbb{R}$ and associated (singular) measured foliation $\mathcal{F}(\rho)$. There is a natural map $\pi: M \rightarrow E$ to the period torus

$$
E=\left(P \otimes_{\mathbb{Z}} \mathbb{R}\right) / P
$$

well-defined up to homotopy. The flux of $(X, \rho)$ is the cohomology class

$$
\operatorname{flux}(X, \rho)=\pi_{*}([\mathcal{F}(\rho)]) \in H_{n-1}(E, \mathbb{R}) .
$$

When it vanishes we say $(X, \rho)$ has zero flux; this means its leaves on average have no homological drift.

Genus two. Now let $L \subset \mathbb{C}$ be a polarized module of rank $2 g, g=2$, such that $L \cap \mathbb{R}$ is Lagrangian.

For each $(X, \omega) \in \mathcal{A}(L)$, we consider the harmonic form $\rho=\operatorname{Im} \omega$ defining $\mathcal{F}(\omega)$. Its periods satisfy

$$
P=\operatorname{Per}(\rho)=\operatorname{Im}(L)=\mathbb{Z} y_{1} \oplus \mathbb{Z} y_{2} \subset \mathbb{R},
$$

where $y_{1}, y_{2} \in \mathbb{R}$ are linearly independent over $\mathbb{Q}$. Thus we can express $\rho$ as a linear combination of harmonic forms with integral periods:

$$
\rho=y_{1} \xi_{1}+y_{2} \xi_{2}
$$

with $\left[\xi_{1}\right],\left[\xi_{2}\right] \in H^{1}(X, \mathbb{Z})$. The period map $\pi: X \rightarrow E \cong \mathbb{R}^{2} / \mathbb{Z}^{2}$ is then obtained by integrating $\xi_{1}$ and $\xi_{2}$, and $(X, \rho)$ has zero flux iff

$$
\operatorname{deg}(X, \rho)=\operatorname{deg}(\pi: X \rightarrow E)=\int_{X} \xi_{1} \wedge \xi_{2}=0 .
$$

(See $[\mathrm{Mc} 7, \S 2]$.$) But this condition means that \left[\xi_{1}\right]$ and $\left[\xi_{2}\right]$ span a Lagrangian subspace of $H^{1}(X, \mathbb{R})$, which is equivalent to the condition that $L \cap \mathbb{R}$ is a Lagrangian submodule of $L$. This shows:

$$
(X, \operatorname{Im} \omega) \text { has zero flux for all }(X, \omega) \in \mathcal{A}(L) \text {. }
$$

By [Mc7, Thm. 7.1], we then obtain the following dichotomy: 
For all $(X, \omega)$ in $\mathcal{A}(L)$, either $\mathcal{F}(\omega)$ is periodic, or it splits into two minimal components, each of genus one.

Upper degree. To refine this dichotomy we introduce the upper degree. A measurable set $A \subset X$ is saturated if it is a union of leaves of $\mathcal{F}(\rho)$. The upper degree is then defined by

$$
f=\operatorname{deg}^{+}(X, \rho)=\sup \int_{A} \xi_{1} \wedge \xi_{2},
$$

where the sup is over all saturated sets.

By [Mc7, Cor 6.4], in the case at hand either

1. $\mathcal{F}(\omega)$ is periodic and $f=0$; or

2. $\mathcal{F}(\omega)$ has two minimal components of genus one, corresponding to a splitting of the form

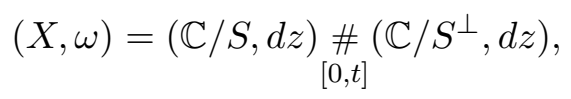

and $f=[\operatorname{Im}(L): \operatorname{Im}(S)]>0$.

In the second case, the maximum in (4.1) is attained when $A$ is one of the minimal components of $\mathcal{F}(\omega)$, and the index $[\operatorname{Im}(L): \operatorname{Im}(S)]$ computes the degree of the map $\pi: A \rightarrow E$.

Here are some additional useful properties of the invariant $f$.

- The upper degree depends only on the topological type of $\mathcal{F}(\omega)$, so it is constant along the leaves of $\mathcal{F}(L)$ (as can be verified from the description just given).

- We have $W(L) \subset \mathcal{A}_{0}(L)$. Indeed, $S \cap \mathbb{R}=(0)$ in equation (4.2), so a double zero is impossible when $f>0$.

- The upper degree strictly increases under limits [Mc7, Theorem 1.3]. That is, along any path $\left(X_{t}, \omega_{t}\right)$ in $\mathcal{A}(L)$ transverse to $\mathcal{F}(L)$, we have

$$
\operatorname{deg}^{+}\left(X_{0}, \omega_{0}\right) \geq 1+\limsup _{t \rightarrow 0} \operatorname{deg}^{+}\left(X_{t}, \omega_{t}\right)
$$

unless $\operatorname{deg}^{+}\left(X_{0}, \omega_{0}\right)=0$.

- Consequently $\mathcal{A}_{0}(L)$ is open and nonempty, and $\bigcup_{f>0} \mathcal{A}_{f}(L)$ is closed. 
- By letting $t$ vary in (4.2) we see that $\bigcup_{f>0} \mathcal{A}_{f}(L)$ is the union of countably many leaves of $\mathcal{F}(L)$, each homeomorphic to $[0, \infty)$ and rooted at a pole $X_{S}(0)$ of $q(L)$.

Periodicity. We now turn to a second characterization of the zero flux condition.

Proposition 4.1 The periodic locus satisfies $\emptyset \neq \mathcal{A}_{0}(L) \neq \mathcal{A}(L)$ iff $L \cap \mathbb{R}$ is Lagrangian.

Proof. Suppose $\emptyset \neq \mathcal{A}_{0}(L) \neq \mathcal{A}(L)$. Then there is a form $(X, \omega) \in \mathcal{A}(L)$ such that $\mathcal{F}(\omega)$ is periodic. The cohomology class $\rho=[\operatorname{Im} \omega] \in H^{1}(X, \mathbb{R})$ has the form $\rho=\sum_{1}^{s} a_{i}\left[C_{i}\right]$, where $\left[C_{i}\right]$ are integral classes Poincaré dual to cylinders of $\mathcal{F}(\omega)$ and $a_{i} \in \mathbb{R}$. We may assume the classes $\left[C_{i}\right]$ are linearly independent over $\mathbb{Q}$; thus $s \leq g=2$. If $s=1$ then $\rho$ is proportional to an integral cohomology class, in which case every form in $\mathcal{A}(L)$ is periodic, contrary to assumption. Thus $s=2$, and therefore $\operatorname{Im}(L)$ and $L \cap \mathbb{R}$ each have rank two. The latter space is Lagrangian, since it has a rational basis satisfying $\left[\omega\left(C_{1}\right), \omega\left(C_{2}\right)\right]=C_{1} \cdot C_{2}=0$. Cf. [Mc7, Cor. 3.2].

The converse will follow from Propositions 4.2 and 4.3 below.

Positive bases. Our next task is to describe $\mathcal{A}_{0}(L)$.

Assume $L \cap \mathbb{R}$ is Lagrangian. Then the symplectic pairing gives an isomorphism

$$
\operatorname{Hom}(L \cap \mathbb{R}, \mathbb{Z}) \cong \operatorname{Im}(L)=\{\operatorname{Im}(\lambda): \lambda \in L\}
$$

Any integral basis $\left(a_{1}, a_{2}\right)$ for $L \cap \mathbb{R}$ determines a dual basis $\left(y_{1}, y_{2}\right)$ for $\operatorname{Im}(L)$, and vice-versa.

We say $\left(a_{1}, a_{2}\right)$ is a positive basis if the real numbers $a_{1}, a_{2}, y_{1}$ and $y_{2}$ are all positive. A positive basis $\left(y_{1}, y_{2}\right)$ for $\operatorname{Im}(L)$ is defined similarly. A positive basis can always be lifted to a symplectic basis $\left(a_{i}, b_{i}\right)$ for $L$ with $\operatorname{Im}\left(b_{i}\right)=y_{i}$, but the pair $\left(b_{1}, b_{2}\right)$ is only well-defined modulo $\mathbb{Z}\left(a_{1}, 0\right) \oplus \mathbb{Z}\left(0, a_{2}\right) \oplus \mathbb{Z}\left(a_{2}, a_{1}\right)$.

The Farey triangulation. The bases for $L \cap \mathbb{R} \cong \mathbb{Z}^{2}$ can be identified with matrices $\left(\begin{array}{ll}a & b \\ c & d\end{array}\right)$, and hence with the Farey geodesics in the hyperbolic plane, which join rationals $a / b$ and $c / d$ with $a d-b c=1$ (see Figure 5 ). The positive bases correspond to those Farey geodesics that separate a pair of distinct, irrational points $\xi_{1}<0<\xi_{2}$ determined by $L \cap \mathbb{R}$ and $\operatorname{Im}(L)$. Thus the positive bases for $L \cap \mathbb{R}$ form an infinite, linearly ordered set. 


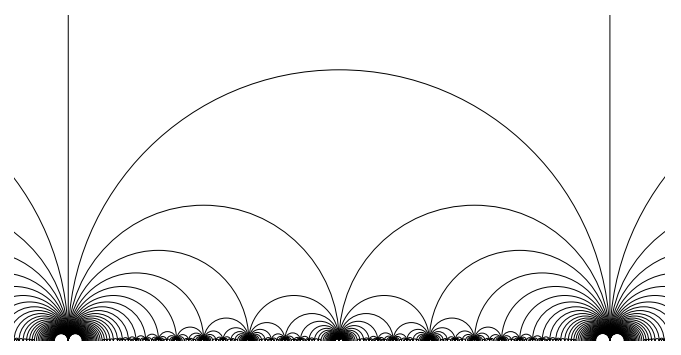

Figure 5. The Farey triangulation, from $0 / 1$ to $1 / 1$.

More concretely, suppose $\left(a_{1}, a_{2}\right)$ and $\left(y_{1}, y_{2}\right)$ are a dual pair of positive bases for $L \cap \mathbb{R}$ and $\operatorname{Im}(L)$. Order the indices so that $a_{1}>a_{2}$. Then the operation

$$
\left(a_{1}, a_{2}\right),\left(y_{1}, y_{2}\right) \rightarrow\left(a_{1}-a_{2}, a_{2}\right),\left(y_{1}, y_{2}+y_{1}\right)
$$

yields a new pair of dual positive bases, with $a_{1}+a_{2}$ smaller. Repeating this operation gives a sequence of bases with $a_{1}+a_{2} \rightarrow 0$; reversing the roles of $a_{i}$ and $y_{i}$, we obtain a sequence with $a_{1}+a_{2} \rightarrow \infty$. These two sequences give all the positive bases for $L \cap \mathbb{R}$.

The spectrum. The spectrum of $L$ is the discrete set of positive numbers

$$
\operatorname{Spec}(L)=\left\{y_{1}+y_{2}:\left(y_{1}, y_{2}\right) \text { is a positive basis for } \operatorname{Im}(L)\right\} \text {. }
$$

Each point $y \in \operatorname{Spec}(L)$ is realized by a unique positive basis $\left(y_{1}, y_{2}\right)$ with $y_{1}>y_{2}$. Indeed, if $y^{\prime}<y$ are consecutive points in $\operatorname{Spec}(L)$, then $\left(y_{1}, y_{2}\right)=$ $\left(y^{\prime}, y-y^{\prime}\right)$. Consequently the two points $y$ and $y^{\prime}$ determine the entire infinite set $\operatorname{Spec}(L) \cap(0, y)$, by the Euclidean algorithm. Note also that $1<y / y^{\prime} \leq 2$.

Proposition 4.2 The locus $\mathcal{A}_{f}(L)$ is nonempty for infinitely many $f>0$.

Proof. Start with any positive splitting $L=S \oplus S^{\perp}$ with $S=\mathbb{Z} a_{1} \oplus \mathbb{Z} b_{1}$, $a_{1} \in \mathbb{R}, \operatorname{Im} b_{1}=y_{1} \neq 0$. Then $B=b_{1}^{\perp} \subset \mathbb{C}$ is isomorphic to $\mathbb{Z}^{3}$, so its closure $\bar{B}$ is a subgroup of $\mathbb{C}$ isomorphic to $\mathbb{R}, \mathbb{R} \times \mathbb{Z}$ or $\mathbb{R}^{2}$. Since $a_{1} \notin B, B \cap \mathbb{R}$ is discrete, and therefore $\bar{B} \neq \mathbb{R} \subset \mathbb{C}$. It follows that $\operatorname{Im}(B)$ is dense in $\mathbb{R}$ and has finite index in $\operatorname{Im}(L)$.

For any $b \in B$ we obtain a new unimodular symplectic module $S^{\prime}=$ $\mathbb{Z} a_{1}^{\prime} \oplus \mathbb{Z} b_{1}$ by setting $a_{1}^{\prime}=a_{1}+b$. This module is positive provided

$$
0<\operatorname{det}\left(S^{\prime}\right)=\operatorname{det}(S)+\operatorname{Im}\left(\bar{b} b_{1}\right)<\operatorname{det}(L),
$$


which holds for all $b \in B$ sufficiently small. Note that $\operatorname{Im}\left(S^{\prime}\right)=\left\langle\operatorname{Im} b, \operatorname{Im} b_{1}\right\rangle \subset$ $\operatorname{Im}(L)$. Since $\operatorname{Im}(B)$ is dense in $\mathbb{R}$, we can simultaneously choose $b$ such that $f=\left[\operatorname{Im}\left(S^{\prime}\right): \operatorname{Im}(L)\right]$ is finite and as large as we want; and then $X_{S}(0) \in \mathcal{A}_{f}(L)$.

Height and strips. Whenever the horizontal foliation $\mathcal{F}(\omega)$ is periodic, we have a tiling of $X$ by the closure of its finitely many cylinders $\left(C_{1}, \ldots, C_{s}\right)$. In fact $s=2$ or 3 , since $\operatorname{Im}(L)$ has rank two and $3 g-3=3$. Let $a_{i}$ and $h_{i}$ denote the circumference and height of $C_{i}$. Note that $a_{i} \in L \cap \mathbb{R}$, since it is given by a period of $\omega$. We define the height function

$$
Y: \mathcal{A}_{0}(L) \rightarrow(0, \infty)
$$

by $Y(X, \omega)=\sum_{1}^{s} h_{i}$.

Given $y \in \operatorname{Spec}(L)$, let

$$
R(y)=\{y-h<Y(X, \omega)<y\} \subset \mathcal{A}_{0}(L),
$$

where $y-h \in \operatorname{Spec}(L)$ is the largest point less than $y$. The next result describes $\bar{R}(y)$; see Figure 6 .

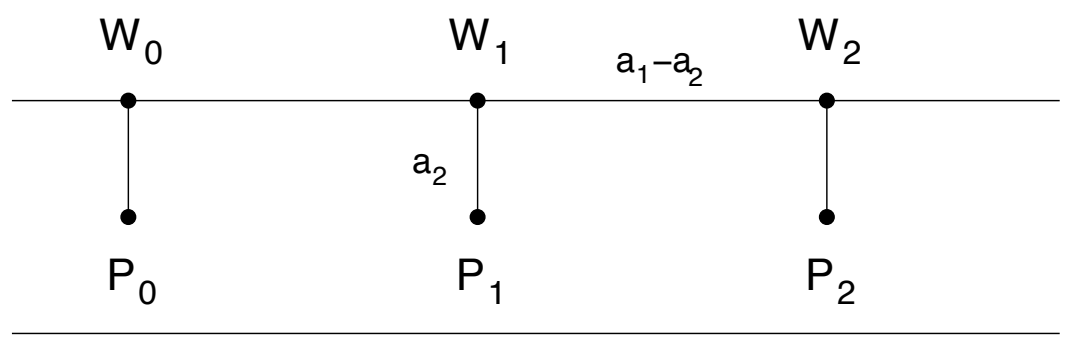

Figure 6 . The region $\bar{R}(y)$ is an infinite flat strip, folded along its upper edge to produce countably many zeros and poles for $q(L)$.

Proposition 4.3 Let $\left(a_{1}, a_{2}\right)$ and $\left(y_{1}, y_{2}\right)$ be the dual pair of positive bases corresponding to $y=y_{1}+y_{2} \in \operatorname{Spec}(L)$, ordered so $a_{1}>a_{2}$. Then:

(i) The level set

$$
\{Y(X, \omega)=y\} \subset \mathcal{A}_{0}(L)
$$

is a single leaf of $\mathcal{F}(q)$, consisting of zeros and poles $W_{m}, P_{m}, m \in \mathbb{Z}$, joined together by segments with lengths

$$
\left|\left[W_{m}, P_{m}\right]\right|=a_{2} \quad \text { and } \quad\left|\left[W_{m}, W_{m+1}\right]\right|=a_{1}-a_{2}
$$

in the metric $|q(L)|$. The poles $P_{m}$ lie in the interior of $\bar{R}(y)$. 
(ii) There is a conformal isomorphism $R(y) \cong\left\{z: 0<\operatorname{Im}(z)<\min \left(y_{1}, y_{2}\right)\right\}$ sending $q(L)$ to $d z^{2}$.

The proof will also show:

Corollary 4.4 The foliation $\mathcal{F}(\omega)$ has two cylinders if $Y(X, \omega) \in \operatorname{Spec}(L)$, and otherwise three.

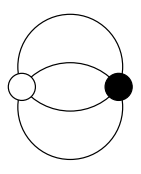

(a)

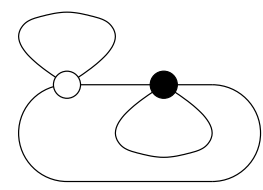

(b)
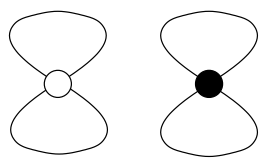

(c)

Figure 7. Ribbon graphs for genus 2, with two or three cylinders.

Proof of Proposition 4.3. As a preliminary, extend $\left(a_{1}, a_{2}\right)$ to a symplectic basis for $L$ by choosing $\left(b_{1}, b_{2}\right)$ with $\left[a_{i}, b_{j}\right]=\delta_{i j}$ and $\left[b_{1}, b_{2}\right]=0$. Then $y_{i}=\operatorname{Im} b_{i}$, and we have a positive splitting

$$
L=\left(\mathbb{Z} a_{1} \oplus \mathbb{Z} b_{1}\right) \oplus\left(\mathbb{Z} a_{2} \oplus \mathbb{Z} b_{2}\right)=S_{0} \oplus S_{0}^{\perp} .
$$

Given $m \in \mathbb{Z}$, we may replace $\left(b_{1}, b_{2}\right)$ with $\left(b_{1}+m a_{2}, b_{2}+m a_{1}\right)$ to obtain another splitting $L=S_{m} \oplus S_{m}^{\perp}$. All positive splittings such that $\operatorname{Im}(S)=\mathbb{Z} y_{1}$ and $\operatorname{Im}\left(S^{\perp}\right)=\mathbb{Z} y_{2}$ are obtained in this way.

We now prove part (i). Let $P_{m}=X_{S_{m}}(0)$ and let $W_{m}=X_{S_{m}}\left(a_{2}\right)$ (recall $\left.a_{2}<a_{1}\right)$. Then $Y\left(P_{m}\right)=Y\left(W_{m}\right)=y$. In fact, all zeros and poles of $q(L)$ along the level set $Y=y$ have this form, since each gives a splitting of type above. Clearly the segment $\left[P_{m}, W_{m}\right]=X_{S_{m}}\left(\left[0, a_{2}\right]\right)$ connecting these points lies along a leaf of $\mathcal{F}(L)$. Along this segment, the saddle connections of $\mathcal{F}(\omega)$ form a graph of the type shown in Figure $7(\mathrm{a})$.

There is a similar segment $\left[W_{m}, W_{m+1}\right]$ of length $a_{1}-a_{2}$, along which the saddle connections have the type shown in Figure 7(b). The behavior of the forms along this path is indicated in Figure 8.

Every two cylinder ribbon graph is of type (a) or (b) (cf. [Mc4, §7]), so to complete the proof we need only show there are no three cylinder forms with $Y(X, \omega)=y$. This will also establish Corollary 4.4.

To this end, consider a 3 -cylinder form with $Y(X, \omega) \in\left(y_{1}, y\right]$, where we have reindexed so that $y_{1}>y_{2}$. It is straightforward to check that the circumferences and heights of its cylinders $\left(C_{1}, C_{2}, C_{3}\right)$ are given by $\left(a_{1}, a_{2}, a_{1}+\right.$ 
$\left.a_{2}\right)$ and $\left(y_{1}-h, y_{2}-h, h\right)$ for some $h \in\left(0, y_{2}\right)$. Thus $Y(X, \omega)$ eq $y_{1}+y_{2}-h$, while $y=y_{1}+y_{2}$.

To prove part (ii), choose a path $\delta$ in the cylinder of height $h$ joining the two different zeros of $\omega$, and let $z=\int_{\delta} \omega$. By shrinking $z$ to zero, we obtain some pole with $Y\left(P_{m}\right)=y$. It follows that $(X, \omega)=X_{S_{m}}(z)$, with $z \in U=\left\{0<\operatorname{Im}(z)<y_{2}\right\}$. Similarly, we have $X_{S_{m}}(U) \subset R(y)$. It is readily verified that this region does not depend on $m$, and thus $R(y) \cong U$ with $q(L)=d z^{2}$.

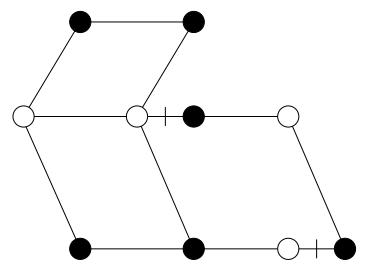

Figure 8. A path from $W_{m}$ to $W_{m+1}$. The two marked edges are identified; all others are glued together by vertical or horizontal translations.

Proof of Theorems 1.2, 1.3 and 1.4. We have seen that $\mathcal{A}_{0}(L)$ is tiled by a sequence of rectangular strips $\bar{R}(y): y \in \operatorname{Spec}(L)$; thus it is a topological disk. By construction, $Y$ sends this tiling of $\mathcal{A}_{0}(L)$ to the tiling of $(0, \infty)$ by intervals with endpoints in $\operatorname{Spec}(L)$. The fact that $W(L) \subset \mathcal{A}_{0}(L)$ and the description of $\bigcup_{f>0} \mathcal{A}_{f}(L)$ are contained in our preliminary remarks on properties of the upper degree.

Cohomological invariants. Let $(X, \rho)$ be a nonzero harmonic form on a Riemann surface $X$. As in [Mc7], the content

$$
C(\rho) \subset H^{1}(X, \mathbb{R})
$$

is defined as the convex hull of the cohomology classes $[\rho \mid A]$, where $A$ ranges over all saturated subsets of $X$ (measurable sets which are unions of leaves of $\mathcal{F}(\rho))$. If $\mathcal{F}(\rho)$ is periodic, with cylinders $C_{i}$ of height $h_{i}$ whose Poincaré duals we denote by $\left[C_{i}\right]$, then

$$
C(\rho)=\left\{\sum a_{i}\left[C_{i}\right]: a_{i} \in\left[0, h_{i}\right]\right\} .
$$

Now let $\rho=\operatorname{Im} \omega$, where $(X, \omega) \in \mathcal{A}(L)$. The invariant $C(\operatorname{Im} \omega)$ depends only on the topological dynamics of $\mathcal{F}(\omega)$, so it is clearly constant along the 
leaves of $\mathcal{F}(L)$. We can now show that the level sets of $C(\operatorname{Im} \omega) \subset \operatorname{Hom}(L, \mathbb{R})$ coincide with the leaves of $\mathcal{F}(L)$.

Proof of Theorem 1.5. Let $\rho=\operatorname{Im} \omega$. Suppose $\mathcal{F}(\omega)$ is periodic with $y=Y(X, \omega) \in \operatorname{Spec}(L)$, and let $\left(a_{1}, a_{2}\right)$ and $\left(y_{1}, y_{2}\right)$ be the corresponding dual pair of positive bases. By Poincaré duality, these cylinders give the classes

$$
a_{1} \otimes y_{1}, a_{2} \otimes y_{2} \in L \otimes_{\mathbb{Z}} \mathbb{R} \cong H_{1}(X, \mathbb{R}) \cong H^{1}(X, \mathbb{R}) .
$$

The vertices of $C(\rho)$ are these two classes, their sum, and 0. Since $a_{1}$ and $a_{2}$ are integral classes, the real numbers $y_{1}$ and $y_{2}$ can be read off from the quadrilateral $C(\rho)$. If $Y(X, \omega) \notin \operatorname{Spec}(L)$ then we have 3 cylinders, and $C(\rho)$ is a hexagon from which we can read off the heights $y_{1}-h, y_{2}-h$ and $h$. So in either case $C(\rho)$ determines the value of $Y(X, \omega)$, which in turn defines a single leaf of $\mathcal{F}(L)$.

Now suppose $\mathcal{F}(\omega)$ has two minimal components, each of genus one. Then $\mathcal{F}(\omega)$ determines a splitting $L=S \oplus S^{\perp}$. The projections of $L$ to $\operatorname{Im}(S)$ and $\operatorname{Im}\left(S^{\perp}\right)$ determine cohomology classes such that $\rho=\rho_{1}+\rho_{2}$, and $C(\rho)$ is the square with vertices $\left(0, \rho_{1}, \rho_{2}, \rho\right)$. Such a square cannot arise in the periodic case, because its vertices are not multiples of integral cohomology classes. From $C(\rho)$ we can recover $S$ as $\operatorname{Ker} \rho_{1}$, and then $(X, \omega)$ belongs to the unique leaf of $\mathcal{F}(L)$ through $X_{S}(0)$.

Since local changes in the relative periods of $(X, \omega)$ can always be constructed via Schiffer variations [Mc6], we have:

Corollary 4.5 Two forms have the same cohomological invariant $C(\operatorname{Im} \omega)$ iff they are related by a sequence of Schiffer variations along the leaves of $\mathcal{F}(\omega)$.

In fact a single variation will suffice, provided the zeros of $\omega$ lie on different leaves of $\mathcal{F}(\omega)$.

\section{$5 \quad$ Hilbert modular surfaces}

In this section we continue the study of $\mathcal{A}(L)$ in the case of eigenforms for real multiplication. In this case $\mathcal{A}(L)$ can be regarded as a slice of a Hilbert modular $X_{D}$, and the zeros and poles of $q(L)$ are slices of algebraic curves $W_{D}$ and $P_{D}$ on this surface. We begin with background and then establish:

Theorem 5.1 Suppose the eigenvalues of $\gamma \in \operatorname{SL}\left(\mathcal{O}_{D} \oplus \mathcal{O}_{D}^{\vee}\right)$ are units in $\mathcal{O}_{D}$ other than \pm 1 . Then $\gamma$ stabilizes at most one component of $\widetilde{P_{D}}$. 
This result will play a pivotal role in the proof of Theorems 1.8 and 1.9 on the topology of the leaves of $\mathcal{F}(L)$. The proof uses positivity of the form $\operatorname{Tr}\left(a a^{\dagger}\right)$ on the endomorphism algebra of an Abelian variety (cf. [BL, 5.1.8]).

We also describe the theta function formula for $q(L)$ from [MZ].

Closed orbits and real multiplication. By [Mc4, Thms. 1.2 and 5.1] we have:

Proposition 5.2 The following conditions are equivalent.

1. The locus $\mathrm{SL}_{2}(\mathbb{R}) \cdot \mathcal{A}(L)$ is a closed subset of $\Omega \overline{\mathcal{M}}_{g}$.

2. The leaf $\mathcal{A}(L)$ is contained in the locus of eigenforms for real multiplication.

3. The trace field of $\mathrm{SL}_{2}(\mathbb{R})^{L}$ is larger than $\mathbb{Q}$.

4. The ring

$$
\operatorname{End}(L)=\{\lambda \in \mathbb{R}: \lambda L \subset L \text { and }[\lambda x, y]=[x, \lambda y] \text { for all } x, y \in L\}
$$

is a real quadratic order $\mathcal{O}_{D}$.

We wish to study $\mathcal{A}(L)$ under the equivalent conditions above.

Lattices and orders. Let $D>0$ be a nonsquare integer congruent to 0 or $1 \bmod 4$. Then $D$ determines a real quadratic order

$$
\mathcal{O}_{D} \subset K=\mathbb{Q}(\sqrt{D}) \subset \mathbb{R}
$$

of discriminant $D$, with a fixed embedding into $\mathbb{R}$. The Galois involution of $K / \mathbb{Q}$ will be denoted by $x \mapsto x^{\prime}$, and the norm and trace by $N(x)$ and $\operatorname{Tr}(x)$. We can write $\mathcal{O}_{D}=\mathbb{Z}[\xi]$, where

$$
\xi=\frac{e+\sqrt{D}}{2}
$$

and $e \in\{0,1\}$ has the same parity as $D$. The inverse different of $\mathcal{O}_{D}$ is the fractional ideal $\mathcal{O}_{D}^{\vee}=(1 / \sqrt{D})$. The module $\mathcal{O}^{D} \oplus \mathcal{O}_{D}^{\vee}$ carries a natural symplectic form, given by

$$
[(a, b),(c, d)]=\operatorname{Tr}(a d-b c) .
$$

Hilbert modular surfaces. Each point $\tau=\left(\tau_{1}, \tau_{2}\right) \in \mathbb{H} \times \mathbb{H}$ determines a polarized lattice

$$
\mathcal{O}_{D} \oplus \mathcal{O}^{\vee} \tau \subset \mathbb{C}^{2}
$$


where $a+b \tau=\left(a+b \tau_{1}, a^{\prime}+b^{\prime} \tau_{2}\right)$. The Hilbert modular surface

$$
X_{D}=\mathbb{H} \times \mathbb{H} / \mathrm{SL}_{2}\left(\mathcal{O}_{D} \oplus \mathcal{O}_{D}^{\vee}\right)
$$

is a degree two branched cover of the locus of all Abelian varieties with real multiplication by $\mathcal{O}_{D}$, via the map

$$
\tau \mapsto \mathbb{C}^{2} / \mathcal{O}_{D} \oplus \mathcal{O}^{\vee} \tau
$$

See e.g. [vG], [Mc5]. Projection of the period lattice to its first factor gives a polarized module

$$
L=\mathcal{O}_{D} \oplus \mathcal{O}^{\vee} \tau_{1} \subset \mathbb{C} .
$$

The definitions readily imply:

Theorem 5.3 For each $\tau_{1} \in \mathbb{H}$ we have a natural isomorphism

$$
\left\{\tau_{1}\right\} \times \mathbb{H} \cong \mathcal{A}(L)
$$

sending $\tau=\left(\tau_{1}, \tau_{2}\right)$ to $(X, \omega)$, where

$$
\operatorname{Jac}(X)=\mathbb{C}^{2} / \mathcal{O}_{D} \oplus \mathcal{O}^{\vee} \tau
$$

and $\omega=d z_{1} \in \Omega(\operatorname{Jac}(X)) \cong \Omega(X)$ is the associated eigenform for real multiplication.

The Weierstrass curve $\boldsymbol{W}_{\boldsymbol{D}}$. The map $\tau \mapsto(X, \omega)$ descends to a welldefined embedding $X_{D} \rightarrow \mathbb{P} \Omega \overline{\mathcal{M}}_{g}$. The locus of eigenforms with double zeros gives the Weierstrass curve $W_{D} \subset X_{D}$, which projects to a Teichmüller curve in $\mathcal{M}_{2}$. It is know that $W_{D}$ has two components when $D=1 \bmod 8$, and is otherwise connected [Mc2, §1].

The nodal locus $\boldsymbol{P}_{\boldsymbol{D}}$. The locus of eigenforms that live on nodal curves defines a curve $P_{D} \subset X_{D}$, disjoint from $W_{D}$, which maps locally isometrically to $\mathcal{M}_{1} \times \mathcal{M}_{1}$. The number of components of $P_{D}$ is given by the number of solutions to $D=e^{2}+4 \ell m$ with $\ell, m>0, e \geq 0$ and $\operatorname{gcd}(e, \ell)=1[\mathrm{Mc} 2$, $\S 2]$.

The components of its preimage $\widetilde{P_{D}}$ in $\mathbb{H} \times \mathbb{H}$ are the graphs of the Möbius transformations $\tau_{2}=U\left(\tau_{1}\right)$ of the form

$$
U=\left(\begin{array}{cc}
\mu & b D \\
-a & -\mu^{\prime}
\end{array}\right)
$$


where $\mu \in \mathcal{O}_{D}, a, b \in \mathbb{Z}, N=\operatorname{det}(U)>0$, and $\mu= \pm(e+\sqrt{D}) / 2$ in $\mathcal{O}_{D} /(\sqrt{D})$ satisfies $e^{2}+4 N=D$. See [Mc5, Theorem 4.1].

Stabilizers. The group $\operatorname{SL}\left(\mathcal{O}_{D} \oplus \mathcal{O}_{D}^{\vee}\right)$ permutes the components of $\widetilde{P_{D}}$, sending the graph of $U$ to the graph of $\gamma^{\prime} U \gamma^{-1}$. The stabilizer of any given component is conjugate, in $\mathrm{SL}_{2}(K)$, to a congruence subgroup of $\mathrm{SL}_{2}(\mathbb{Z})$.

In general, an element $\gamma \in \operatorname{SL}\left(\mathcal{O}_{D} \oplus \mathcal{O}_{D}^{\vee}\right)$ can stabilize more than one component of $\widetilde{P_{D}}$. For example, when $D=8$ the matrices $U_{1}=\left(\begin{array}{cc}\sqrt{2} & 0 \\ 0 & \sqrt{2}\end{array}\right)$ and $U_{2}=\left(\begin{array}{cc}5 \sqrt{2} & 8 \\ 6 & 5 \sqrt{2}\end{array}\right)$ are both fixed by $\gamma=\left(\begin{array}{ll}7 & 8 \\ 6 & 7\end{array}\right)$.

Theorem 5.1 asserts that the behavior is different if $\gamma$ is diagonalizable over $K$. Then only one component of $\widetilde{P_{D}}$ can be fixed by $\gamma$ (provided $\gamma \neq \pm I$ ). This fact will play an important role in the sequel.

Proof of Theorem 5.1. Let $\epsilon^{ \pm 1}$ be the eigenvalues of $\gamma$, and let $K^{2}=$ $V_{-} \oplus V_{+}$be the corresponding eigenspace decomposition.

Suppose $\gamma$ stabilizes two components of $\widetilde{P_{D}}$, corresponding to the symplectic splittings $\mathcal{O}_{D} \oplus \mathcal{O}_{D}^{\vee}=S_{i} \oplus S_{i}^{\perp}, i=1,2$. Then $S_{i} \otimes \mathbb{Q}$ can be regarded as the graph of a map $f_{i}: V_{-} \rightarrow V_{+}$commuting with the action of $\gamma$. Such a map is unique up to composition with an element of $\mathbb{Q}(\gamma)=K$. Thus $S_{1}=k S_{2}$ for some $k \in K$. It follows that the ring

$$
A=\left\{\delta \in M_{2}\left(\mathcal{O}_{D} \oplus \mathcal{O}_{D}^{\vee}\right): \delta\left(S_{i}\right)=S_{i} \text { and } \delta \gamma=\gamma \delta\right\}
$$

is independent of $i$; that is, it is uniquely determined by $\gamma$. Note that $A$ is invariant under the Rosati involution (defined by $[\delta x, y]=\left[x, \delta^{\dagger} y\right]$ ).

Now let $\pi_{i} \in A$ be the projection of $S_{i} \oplus S_{i}^{\perp}$ onto its first factor. Then $\pi_{i}^{\perp}=\pi_{i}$, and $\pi_{i}$ commutes with $\gamma$. Since $\gamma$ acts irreducibly on $S_{1}$, with respect the splitting $S_{1} \oplus S_{1}^{\perp}$ we have

$$
\pi_{2}=\left(\begin{array}{cc}
a I & b \\
b^{\dagger} & c I
\end{array}\right)
$$

for some $a, c \in \mathbb{Z}$. Since $\pi_{2} \circ \pi_{2}=\pi_{2}$, we also have $a^{2}+b b^{\dagger}=a$. But $\operatorname{Tr}\left(b b^{\dagger}\right) \geq 0$, so $b=0$ and $a=0$ or 1 . It follows that $\pi_{2}=\pi_{1}$ or $I-\pi_{1}$, and hence the two splittings are the same.

Theta functions. We conclude by describing the formula used to draw $\mathcal{F}\left(L_{D}\right)$ in Figures 2, 4 and 12. The classical theta function associated to $\tau \in \mathbb{H} \times \mathbb{H}$ is defined for $z=\left(z_{1}, z_{2}\right) \in \mathbb{C}^{2}$ by

$$
\vartheta(\tau, z)=\sum_{n \in \mathcal{O}_{D}^{\vee}} q^{n^{2}} \zeta^{n}
$$


where

$$
\zeta^{a}=\zeta_{1}^{a} \zeta_{2}^{a^{\prime}}=\exp \left(2 \pi i a z_{1}\right) \exp \left(2 \pi i a^{\prime} z_{2}\right)
$$

and similarly

$$
q^{a}=q_{1}^{a} q_{2}^{a^{\prime}}=\exp \left(\pi i a \tau_{1}\right) \exp \left(\pi i a^{\prime} \tau_{2}\right)
$$

for any $a \in K$. The zero set of $\vartheta(\tau, z)$ in $\mathbb{C}^{2}$ covers the theta divisor

$$
X \subset \mathbb{C}^{2} /\left(\mathcal{O}_{D} \oplus \mathcal{O}_{D}^{\vee} \tau\right) .
$$

In the present setting $X$ is a smooth curve of genus two, and the ambient complex torus can be identified with the Jacobian of $X$.

Let $M=\mathcal{O}_{D} \oplus \mathcal{O}_{D}^{\vee}$. Given $(a, b) \in M \otimes \mathbb{Q}$, let

$$
\vartheta_{(a, b)}(\tau, z)=\exp (2 \pi i \operatorname{Tr}(a b)) q^{b^{2}} \zeta^{b} \vartheta(\tau, z+a+b \tau) .
$$

We have $\vartheta_{m}(\tau, z)=\vartheta(\tau, z)$ for all $m \in M$.

The 16 points $[m] \in \frac{1}{2} M / M$ define spin structures on $X$. The 10 even spin structures are those which satisfy

$$
\sum 4\left[m, a_{i}\right]\left[m, b_{i}\right]=0 \bmod 2
$$

with respect to a symplectic basis for $M$; the other 6 are odd. The odd spin structures coincide with Weierstrass points on $X$, and the even ones give the possible locations for a node. Choosing a representative $m \in \frac{1}{2} M$ for each spin structure, we define a meromorphic form on $\mathbb{H} \times \mathbb{H}$ by

$$
Q=\left(\prod_{m \text { odd }} \frac{d \vartheta_{m}}{d z_{2}}(\tau, 0) / \prod_{m \text { even }} \vartheta_{m}(\tau, 0)\right) d \tau_{1}^{-1} d \tau_{2}^{2} .
$$

This form is invariant under $\operatorname{SL}\left(\mathcal{O}_{D} \oplus \mathcal{O}_{D}^{\vee}\right)$, and we have:

Theorem 5.4 (Möller-Zagier) The divisor of $Q$ on $X_{D}$ is given by $(Q)=$ $W_{D}-P_{D}$, and its restriction to a slice

$$
\left\{\tau_{1}\right\} \times \mathbb{H} \cong \mathcal{A}(L)
$$

gives a nonzero multiple of the relative period form $q(L), L=\mathcal{O}_{D} \oplus \mathcal{O}_{D}^{\vee} \tau$.

For more on the differential $Q$, see $[\mathrm{Mc} 5, \S 8]$ and $[\mathrm{Ba}, \S 10]$. A general reference for $\vartheta$-functions is [Mum]. 


\section{Dynamics of $\mathrm{SL}_{2}\left(\mathcal{O}_{D}\right)$}

In this section we continue the study of eigenforms for real multiplication, with the normalization

$$
L_{D}=\mathcal{O}_{D} \oplus \mathcal{O}_{D} i
$$

(corresponding to $\tau_{1}=i \sqrt{D}$ ). The symplectic form on $L_{D}$ is given by $[a+i b, c+i d]=\operatorname{Tr}_{\mathbb{Q}}^{K}\left(D^{-1 / 2}(a d-b c)\right)$, and we have

$$
\mathrm{SL}_{2}(\mathbb{R})^{L_{D}}=\mathrm{SL}_{2}\left(\mathcal{O}_{D}\right) .
$$

We establish Theorem 1.6 on the orbits of $\mathrm{SL}_{2}\left(\mathcal{O}_{D}\right)$, and analyze the geometry of $\mathcal{A}_{0}(L)$.

The standard splitting. As in $\S 5$, let $\xi=(e+\sqrt{D}) / 2$ where $e \in\{0,1\}$ has the same parity as $D$; then $\mathcal{O}_{D}=\mathbb{Z}[\xi]$. A symplectic basis for $L_{D}$ is given by $\left(a_{1}, b_{1}\right)=\left(1,-\xi^{\prime} i\right)$ and $\left(a_{2}, b_{2}\right)=(\xi, i)$. This basis defines the standard splitting

$$
L_{D}=\left(\mathbb{Z} a_{1} \oplus \mathbb{Z} b_{1}\right) \oplus\left(\mathbb{Z} a_{2} \oplus \mathbb{Z} b_{2}\right)=S \oplus S^{\perp} .
$$

Since $a_{i}, \operatorname{Im}\left(b_{i}\right)>0$, this splitting is positive, and we have

$$
\operatorname{det}\left(L_{D}\right)=\operatorname{Im}\left(\bar{a}_{1} b_{1}\right)+\operatorname{Im}\left(\bar{a}_{2} b_{2}\right)=\xi-\xi^{\prime}=\sqrt{D} .
$$
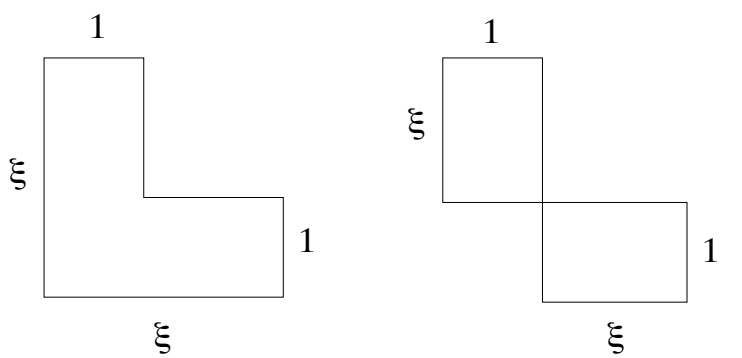

Figure 9. Forms in $W\left(L_{D}\right)$ and $P\left(L_{D}\right)$ with rotational symmetry.

Complex multiplication. When $D=1 \bmod 4$ we have $\left(a_{1}, b_{1}\right)=(1, \xi-1)$, and thus the form $(X, \omega)$ obtained from the $L$-shaped polygon in Figure 9 lies in $W\left(L_{D}\right)$. Because of its 4 -fold symmetry, $(X, \omega)$ is an eigenform for complex multiplication by $\mathcal{O}_{D}[i]$. This point is fixed by the elliptic element $\tau \mapsto-D / \tau$ in $\operatorname{SL}\left(\mathcal{O}_{D} \oplus \mathcal{O}_{D}^{\vee}\right)$, so in fact $\tau_{2}(X, \omega)=i \sqrt{D}$. This is one of the few points in $W\left(L_{D}\right)$ whose coordinate $\tau_{2} \in \mathbb{H}$ can be expressed in closed form. (A complete classification of elliptic points on $W_{D}$ is given in $[\mathrm{Mu}]$.) 
When $D=0 \bmod 4$, the same fixed point lies in $P\left(L_{D}\right)$ and comes from the pair of rectangles at the right in Figure 9. (In fact all the points of $P\left(L_{D}\right)$ can be computed directly for any value of $\tau_{1}$, using equation (5.2).) Units and periodicity. Let $\epsilon>1$ denote the fundamental unit for $\mathcal{O}_{D}$.

The geodesic in $\mathbb{H}$ with endpoints $\xi$ and $\xi^{\prime}$ is stabilized by a matrix $A \in \mathrm{SL}_{2}(\mathbb{Z})$ with eigenvalues $\epsilon^{ \pm 1}$. Similarly, $\operatorname{Spec}\left(L_{D}\right)$ is invariant under multiplication by $\epsilon$; and if we let $\left[c_{1}, \ldots, c_{n}\right]$ denote the periodic part of the continued fraction expansion of $\xi$, we may use the Farey triangulation to verify:

Proposition 6.1 The number of points in $\operatorname{Spec}\left(L_{D}\right) /\langle\epsilon\rangle$ is given by $N=$ $c_{1}+\cdots+c_{n}$, where $\left[c_{1}, \ldots, c_{n}\right]$ is the periodic part of the continued fraction expansion of $\xi$.

Thus the strips $\bar{R}(y), y \in \operatorname{Spec}\left(L_{D}\right)$ that cover $\mathcal{A}_{0}\left(L_{D}\right)$ have essentially $N$ different types.

Theorem 6.2 The discrete sets $P\left(L_{D}\right)$ and $W\left(L_{D}\right)$ fall into finitely many orbits under the action of $\mathrm{SL}_{2}\left(\mathcal{O}_{D}\right)$ on $\mathcal{A}\left(L_{D}\right)$. Every other orbit of $\mathrm{SL}_{2}\left(\mathcal{O}_{D}\right)$ is dense, with the exception of the decagon orbit in the case $D=5$.

Proof. These orbits correspond to the finitely many components of the curves $W_{D}$ and $P_{D}$. The second statement follows from the analysis of orbit closures for $\mathrm{SL}_{2}(\mathbb{R})$ acting on $\Omega \mathcal{M}_{g}$ [Mc4], together with the classification of Teichmüller curves in genus two [Mc3]. (This reference also describes the decagon form.)

Foliations and the affine group. Observe that $\mathcal{O}_{D}=L_{D} \cap \mathbb{R}$ is a Lagrangian subspace of $L_{D}$. Thus we have a stratification $\mathcal{A}\left(L_{D}\right)=\bigcup_{f \geq 0} \mathcal{A}_{f}\left(L_{D}\right)$ as in $\S 4$.

The affine group of upper triangular matrices in $\mathrm{SL}_{2}\left(\mathcal{O}_{D}\right)$ is given in terms of the fundamental unit $\epsilon$ by

$$
\mathrm{AN}_{2}\left(\mathcal{O}_{D}\right)=\left\{\gamma= \pm\left(\begin{array}{cc}
\epsilon^{-n} & b \\
0 & \epsilon^{n}
\end{array}\right): n \in \mathbb{Z} \text { and } b \in \mathcal{O}_{D}\right\}
$$

This group preserves the leaves of the foliation $\mathcal{F}\left(L_{D}\right)$ and the strata $\mathcal{A}_{f}\left(L_{D}\right)$, and we have

$$
Y(\gamma \cdot(X, \omega))=\epsilon^{n} Y(X, \omega)
$$


in particular, the unipotent subgroup $N_{2}\left(\mathcal{O}_{D}\right) \subset \mathrm{AN}_{2}\left(\mathcal{O}_{D}\right)$ preserves the level sets of $Y$.

Each of the sets $P_{f}\left(L_{D}\right)=P\left(L_{D}\right) \cap \mathcal{A}_{f}(L)$ is invariant under $\operatorname{AN}_{2}\left(\mathcal{O}_{D}\right)$. The natural bijection $P_{0}\left(L_{D}\right) \rightarrow W_{D}$, obtained by following the leaves of $\mathcal{F}(q)$, descends to a bijection

$$
W\left(L_{D}\right) / \mathrm{AN}_{2}\left(\mathcal{O}_{D}\right) \cong P_{0}\left(L_{D}\right) / \mathrm{AN}_{2}\left(\mathcal{O}_{D}\right)
$$

Using the fact that $N_{2}\left(\mathcal{O}_{D}\right)$ acts nontrivially on each strip, it is easy to see that both sets above are finite. In fact:

Proposition 6.3 The number of cusps of $W_{D}$ is an upper bound for the number of orbits of $\mathrm{AN}_{2}\left(\mathcal{O}_{D}\right)$ acting on $W\left(L_{D}\right)$.

Proof. Suppose for simplicity that $W_{D}$ is connected. Let $\Gamma \subset \mathrm{SL}_{2}\left(\mathcal{O}_{D}\right)$ be the stabilizer of a point $W_{0} \in W\left(L_{D}\right)$. Then $W\left(L_{D}\right)$ may be identified with $\mathrm{SL}_{2}\left(\mathcal{O}_{D}\right) / \Gamma$, and its quotient by $\mathrm{AN}_{2}\left(\mathcal{O}_{D}\right)$ may be identified with $C / \Gamma$, where

$$
C=\mathrm{AN}_{2}\left(\mathcal{O}_{D}\right) \backslash \mathrm{SL}_{2}\left(\mathcal{O}_{D}\right) \hookrightarrow \mathrm{AN}_{2}(\mathbb{R}) \backslash \mathrm{SL}_{2}(\mathbb{R}) \cong \mathbb{P}^{1}(\mathbb{R})
$$

Since the horizontal foliation of any form in $W\left(L_{D}\right)$ is periodic, the stabilizer in $\Gamma$ of any point in $C$ is unipotent. Hence $C / \Gamma$ corresponds to a subset of the cusps of $W_{D}=\mathbb{H} / \Gamma$. Similar reasoning applies if $W_{D}$ has more than one component.

Proposition 6.4 For each $f>0$, the set $P_{f}\left(L_{D}\right)$ falls into finitely many orbits under the action of $\mathrm{N}_{2}\left(\mathcal{O}_{D}\right)$.

Proof. A point in $P\left(L_{D}\right)$ is determined by the associated positive splitting $L_{D}=S \oplus S^{\perp}$. Thus it suffices to show there are only finitely such splittings with $\left[\operatorname{Im}(S): \operatorname{Im}\left(L_{D}\right)\right]=d$, up to the action of $N_{2}\left(\mathcal{O}_{D}\right)$.

Clearly there are only finitely many possibilities for $\operatorname{Im}(S)$. Fix one such, with integral basis $y_{1}, y_{2}$. We may assume $S$ has a basis with $\operatorname{Im}\left(a_{1}\right)=$ $y_{1}, \operatorname{Im}\left(b_{1}\right)=y_{2}$ and $\left[a_{1}, b_{1}\right]=1$. The orbit of $a_{1}$ under $N_{2}\left(\mathcal{O}_{D}\right)$ is $a_{1}+y_{1} \mathcal{O}_{D}$, which has finite index in the set $a_{1}+\mathcal{O}_{D}$ of all solutions to $\operatorname{Im}(z)=y_{1}$ in $L_{D}$. Thus there are only finitely many possibilities for $a_{1}$ up to the action of $\mathrm{N}_{2}\left(\mathcal{O}_{D}\right)$. 
Now suppose $a_{1}$ is fixed. Then the set of all solutions to the equations $\operatorname{Im} b_{1}=y_{1}$ and $\left[a_{1}, b_{1}\right]=1$ in $L_{D}$ forms a coset of the form $z_{1}+\mathbb{Z} x_{1}$. But the splitting $S \oplus S^{\perp}$ is positive, so $b_{1}=z_{1}+n x_{1}$ must also satisfy

$$
0<\operatorname{Im}\left(\bar{a}_{1} b_{1}\right)=\operatorname{Im}\left(\bar{a}_{1} z_{1}\right)+n x_{1} y_{1}<\operatorname{det}\left(L_{D}\right) .
$$

Thus once $a_{1}$ is fixed, there are only finitely many choices for $b_{1}$ with the required positivity.

Proof of Theorem 1.6: Combine the results above and use the fact that $\mathrm{N}_{2}\left(\mathcal{O}_{D}\right) \subset \mathrm{AN}_{2}\left(\mathcal{O}_{D}\right)$.

Geometry of the periodic stratum. We conclude this section by studying the domain $\mathcal{A}_{0}\left(L_{D}\right) \subset \mathbb{H}$ in the coordinate system $\tau_{2}$.

Proposition 6.5 The imaginary axis $\operatorname{Re} \tau_{2}=0$ lies in $\mathcal{A}_{0}\left(L_{D}\right)$.

Proof. Since $\operatorname{Re}\left(\tau_{1}\right)=0$, every form with $\operatorname{Re}\left(\tau_{2}(X, \omega)\right)=0$ has an anticonformal symmetry preserving the leaves of $\mathcal{F}(\omega)$. Such a symmetry is only compatible with a periodic foliation.

Since $\mathcal{A}_{0}(L)$ is invariant under the dense subgroup $\mathrm{SL}_{2}\left(\mathcal{O}_{D}\right) \subset \mathrm{SL}_{2}(\mathbb{R})$, we have:

Corollary 6.6 There is a dense set of pairs $\left(x_{1}, x_{2}\right) \in \mathbb{R}^{2}$ such that $x_{1}$ can be joined to $x_{2}$ by an arc in $\mathcal{A}_{0}(L) \subset \mathbb{H}$.

Galois conjugate dynamics at the boundary. By [Mc5, Theorem 8.3], for each $\gamma \in \mathrm{SL}_{2}\left(\mathcal{O}_{D}\right)$ the map $f_{\gamma}$ acts on $\partial \mathcal{A}(L) \cong \mathbb{R} \cup\{\infty\}$ by the Galois conjugate Möbius transformations $\gamma^{\prime} \in \mathrm{SL}_{2}(\mathbb{R})$. More precisely, in $\tau_{2}$ coordinates on $\mathbb{R}$ we have

$$
f_{\gamma}(x)=C \gamma^{\prime}(x / C)
$$

for some $C>0$. (In the case at hand, $C=\sqrt{D}$; this scale factor arises from the isomorphism $\operatorname{SL}_{2}\left(\mathcal{O}_{D}\right) \cong \operatorname{SL}\left(\mathcal{O}_{D} \oplus \mathcal{O}_{D}^{\vee}\right)$.)

Using this boundary dynamics, we will show:

Proposition 6.7 Each leaf $H$ of $\mathcal{F}\left(L_{D}\right)$ in $\mathcal{A}_{0}\left(L_{D}\right) \subset \mathbb{H}$ lies within a bounded hyperbolic distance of a horocycle of the form $\operatorname{Im} \tau_{2}=y$, and its ends converge to $\tau_{2}=\infty$. 
Proof. Let $\left\langle\ldots t_{-1}<t_{0}<t_{1} \ldots\right\rangle$ be the ordered set of $t \in \mathcal{O}_{D} \subset \mathbb{R}$ with $\left|t^{\prime}\right| \leq 1$. Since $\left\{\left(t, t^{\prime}\right): t \in \mathcal{O}_{D}\right\}$ gives a lattice in $\mathbb{R}^{2}$, we have $M=\sup \left|t_{n+1}-t_{n}\right|<\infty$. Let $f_{n}: \mathbb{H} \rightarrow \mathbb{H}$ denote the map $f_{\gamma_{n}}$ for

$$
\gamma_{n}=\left(\begin{array}{cc}
1 & t_{n}^{\prime} \\
0 & 1
\end{array}\right) \in \mathrm{SL}_{2}\left(\mathcal{O}_{D}\right)
$$

Since $D_{q} f_{n}=\gamma_{n}$ and $\left|t_{n}^{\prime}\right| \leq 1$, these maps are uniformly quasiconformal. By equation (6.1), the map $f_{n} \mid \mathbb{R}$ agrees a Möbius transformation of the form $T_{n}(z)=z+C t_{n}$, where $C$ is independent of $n$.

Let $H$ be the leaf of $\mathcal{F}\left(L_{D}\right)$ through $Z_{0} \in \mathcal{A}_{0}\left(L_{D}\right)$. Then $H$ is invariant under $N_{2}\left(\mathcal{O}_{D}\right)$, so in particular it is invariant under the maps $f_{n}$ above. It follows readily that $H$ is a quasiarc and its ends converge to $\tau_{2}=\infty$. By properties of quasiconformal maps, we have $d\left(f_{n}\left(Z_{0}\right), T_{n}\left(Z_{0}\right)\right)=O(1)$ in the hyperbolic metric. Since every point on the horocycle $\operatorname{Im} \tau_{2}=\operatorname{Im} Z_{0}$ lies within a bounded distance of a point of the form $T_{n}\left(Z_{0}\right)=Z_{0}+t_{n}$, and the Proposition follows.

We will see that $\mathcal{A}_{0}(L)$ contains a halfplane in Corollary 7.4 below.

Remark: Other ideals. When the class number of $\mathcal{O}_{D}$ is greater than one, not every Lagrangian subspace of $L_{D}$ is equivalent to $\mathcal{O}_{D}$ under the action of $\mathrm{SL}_{2}\left(\mathcal{O}_{D}\right)$. To obtain a complete picture, one should also consider the case where $I \subset \mathcal{O}_{D}$ is an ideal and $L=I \oplus I^{\vee} \tau_{1}$. This is necessary, for example, to see all the cusps of $W_{D}$ in Proposition 6.3.

\section{$7 \quad$ Fixed points and pseudo-Anosov maps}

In this section we relate the action of $\mathrm{SL}_{2}\left(\mathcal{O}_{D}\right)$ on $\mathcal{A}\left(L_{D}\right)$ to the existence of pseudo-Anosov maps, and prove Theorem 1.7. We then use dynamics of $\mathrm{AN}_{2}\left(\mathcal{O}_{D}\right)$ to tame the leaves of $\mathcal{F}\left(L_{D}\right)$, and establish Theorems 1.8 and 1.9 on the behavior of $\partial \mathcal{A}_{0}(L)$.

Realizations. We continue with the assumption $g=2$. Let $\gamma \in \operatorname{Sp}_{2 g}(\mathbb{Z})$ be a symplectic automorphism with largest eigenvalue $\lambda>1$. Suppose $\gamma$ is irreducible; then $\lambda$ has degree 4 over $\mathbb{Q}$, and $\gamma$ commutes with the real quadratic order generated by $\gamma+\gamma^{-1}$ in $\mathrm{M}_{2 g}(\mathbb{Z})$. Thus we can consider $\gamma$ as an element of $\mathrm{SL}_{2}\left(\mathcal{O}_{D}\right)$.

We say $\gamma$ is realized by a pseudo-Anosov map $F: \Sigma_{g} \rightarrow \Sigma_{g}$ if $F$ has multiplier $\lambda$ and $\gamma=F \mid H^{1}\left(\Sigma_{g}\right)$. (In this case the stable and unstable foliations of $F$ must be orientable.) 
Theorem 7.1 The element $\gamma \in \mathrm{SL}_{2}\left(\mathcal{O}_{D}\right)$ is realizable iff the quasiconformal map $f_{\gamma}$ has a fixed point on $\mathcal{A}\left(L_{D}\right)-P\left(L_{D}\right)$.

Proof. The stabilizer of $(X, \omega) \in \mathcal{A}\left(L_{D}\right)$ is simply the affine group $\operatorname{SL}(X, \omega)$. Thus if $\gamma$ has a fixed point, it is realized by a pseudo-Anosov mapping on $X$ with foliations parallel to its eigenvectors in $\mathbb{R}^{2} \cong \mathbb{C}$. (Since $\gamma$ is irreducible, $(X, \omega) \notin P\left(L_{D}\right)$.) The converse is similar.

Proof of Theorem 1.7. Suppose $|\operatorname{Tr}(\gamma)|>2>\left|\operatorname{Tr}(\gamma)^{\prime}\right|$, and identify $\mathcal{A}\left(L_{D}\right)$ with $\mathbb{H}$. By equation $(6.1), f_{\gamma} \mid \partial \mathbb{H}$ has no fixed points since it acts by an elliptic Möbius transformation with trace $\operatorname{Tr}(\gamma)^{\prime}$. Thus $f_{\gamma}$ fixes a point in $\mathbb{H}$ by Brouwer's fixed point theorem.

On the other hand, we have:

Proposition 7.2 If $|\operatorname{Tr}(\gamma)| \leq\left|\operatorname{Tr}(\gamma)^{\prime}\right|$, then $\gamma$ is not realizable.

Proof. If $\gamma$ is realizable then its leading eigenvalue as an element of $\operatorname{Sp}_{2 g}(\mathbb{Z})$ must be simple and agree with the multiplier $\lambda$ of the associated pseudoAnosov map, which is the leading eigenvalue of $\gamma$ as an element of $\mathrm{SL}_{2}\left(\mathcal{O}_{D}\right)$.

The affine group. Next we examine the fixed points of the affine group.

Theorem 7.3 The fixed points of hyperbolic elements in $\mathrm{AN}_{2}\left(\mathcal{O}_{D}\right)$ all lie in $\bigcup_{f>0} P_{f}\left(L_{D}\right)$. A given hyperbolic element has at most one fixed point, and every point in $\bigcup_{f>0} P_{f}\left(L_{D}\right)$ is fixed by at least one hyperbolic element.

Proof. Elements of the affine group can never be realized by orientable pseudo-Anosov mappings, because they satisfy $|\operatorname{Tr}(\gamma)|=\left|\operatorname{Tr}\left(\gamma^{\prime}\right)\right|$. Thus their fixed points lie in $P\left(L_{D}\right)$. Such a fixed point $(X, \omega)$ is unique by Theorem 5.1. The foliation $\mathcal{F}(\omega)$ must be irrational if $\gamma$ is hyperbolic, so $(X, \omega) \in P_{f}\left(L_{D}\right)$ for some $f>0$.

Conversely, any form $(X, \omega) \in \bigcup_{f>0} P_{f}\left(L_{D}\right)$ gives a pair of isogenous tori with foliations $\mathcal{F}(\omega)$ of equal, irrational slope $k \in K$, and is therefore stabilized by some hyperbolic element in $\mathrm{AN}_{2}\left(\mathcal{O}_{D}\right)$. 
Convergence of leaves. Let $P \in P_{f}\left(L_{D}\right), f>0$, be stabilized by the hyperbolic element $\gamma \in \mathrm{AN}_{2}\left(\mathcal{O}_{D}\right)$. We can now show that the leaf of $\mathcal{F}\left(L_{D}\right)$ through $P$ converges to the unique fixed point $x$ of $\gamma$ in $\mathbb{R} \subset \partial \mathcal{A}\left(L_{D}\right)$.

Proof of Theorem 1.8. Let $M \subset \mathcal{A}\left(L_{D}\right)$ be the closure of the leaf through $P$. Then $M$ itself is a union of leaves of $\mathcal{F}\left(L_{D}\right)$. If $M$ is compact, then by equation (4.3) there is a $Z \in M$ where $\operatorname{deg}^{+}(Z)$ is maximized. The same result shows the leaf through $Z$ cannot have any accumulation point in $M$, which is a contradiction since this leaf is isomorphic to $[0, \infty)$.

Thus $M$ accumulates on at least one point $x \in \partial \mathbb{H}$. By Corollary 6.6 and Proposition 6.7 , there is only one such accumulation point and $x \in \mathbb{R}$. Thus $x$ must coincide with the real fixed point of $\gamma$. The leaf through any other pole $Q \in M$ must also converge to $x$. Since the stabilizer of $x$ in $\operatorname{AN}_{2}\left(\mathcal{O}_{D}\right)$ is virtually cyclic, it follows that then $P$ and $Q$ are stabilized by the same hyperbolic element, and hence $P=Q$ by Theorem 7.3. Consequently $M$ contains only one leaf of $\mathcal{F}\left(L_{D}\right)$. By equation (4.3) the leaf cannot accumulate on itself, so it must in fact converge to $x$.

Accessibility. Finally we show that points in $\mathcal{A}_{f}\left(L_{D}\right)$ are inaccessible from $\mathcal{A}_{0}\left(L_{D}\right)$ for all $f \gg 0$.

Proof of Theorem 1.9. Suppose $Z_{0}=X_{S}(0) \in P_{f}\left(L_{D}\right)$, for $f>0$. Recall that $Z_{0}$ is fixed by a hyperbolic element $\gamma \in \mathrm{AN}_{2}\left(\mathcal{O}_{D}\right)$.

Let $s \neq 0$ be one of the nonzero points in $S \cup S^{\perp}$ closest to the origin. Then the path $Z_{t}=X_{S}(t)$ joins $Z_{0}$ to a point $Z_{s}=W \in W(L) \subset \mathcal{A}_{0}(L)$. If this path crosses $\partial \mathcal{A}_{0}\left(L_{D}\right)$, then we can use the action of $\gamma$ to prove that $Z_{0}$ is inaccessible as in Figure 3.

Otherwise, the segment $[0, s] \subset \mathbb{C}$ corresponds to a saddle connection $C$ of $W=(X, \omega)$ crossing each of the two cylinders of $\mathcal{F}(\omega)$ exactly once, so that $\int_{C} \operatorname{Im} \omega=Y(W)$. There are only finitely many such $C$ up to the action of the cyclic group $\mathrm{AN}_{2}\left(\mathcal{O}_{D}\right)^{W}$, and there are only finitely many $\mathrm{AN}_{2}\left(\mathcal{O}_{D}\right)$ orbits in $W\left(L_{D}\right)$, so there are only finitely many $\mathrm{AN}_{2}\left(\mathcal{O}_{D}\right)$ orbits of accessible points in $P\left(L_{D}\right)$.

Since the upper degree is constant on each orbit, we have shown that $P_{f}\left(L_{D}\right)$ is inaccessible for all $f \gg 0$. But once a point in $\mathcal{A}\left(L_{D}\right)$ is inaccessible, so is the whole leaf of $\mathcal{F}\left(L_{D}\right)$ through it, so $\mathcal{A}_{f}\left(L_{D}\right)$ is inaccessible for all $f \gg 0$.

Corollary 7.4 The periodic locus $\mathcal{A}_{0}(L)$ contains a horoball of the form $U=\left\{\operatorname{Im} \tau_{2}>y>0\right\}$. 
Proof. Let $H$ be a leaf of $\mathcal{F}\left(L_{D}\right)$ contained in $\mathcal{A}_{0}\left(L_{D}\right)$. By Proposition 6.7, $H$ lies below a line of the form $\operatorname{Im} \tau_{2}=y$ in $\mathbb{H}$ and converges to infinity. Thus $H$ separates $U$ from the real axis. By the preceding result, any point in $\mathcal{A}\left(L_{D}\right)-\mathcal{A}_{0}\left(L_{D}\right)$ can be joined to the real axis by a path avoiding $H$; thus $U \subset \mathcal{A}_{0}\left(L_{D}\right)$.

\section{The case $D=5$}

In this section we give a more detailed account of the case $D=5$.

Splittings. To begin with, we note that the generator $\xi=(1+\sqrt{5}) / 2$ of $\mathcal{O}_{D}=\mathbb{Z}[\xi]$ coincides with the fundamental unit $\epsilon>1$. It follows easily that

$$
\operatorname{Spec}\left(L_{D}\right)=\epsilon^{\mathbb{Z}}
$$

Note also that $\epsilon^{\prime}=-1 / \epsilon$.

This case is simplified by the fact that $L_{D}$ has only one type of splitting.

Proposition 8.1 For $D=5$, any positive splitting

$$
L_{D}=S \oplus S^{\perp}
$$

satisfies $S^{\perp}=\epsilon^{ \pm 1} S$.

Proof. Consider a positive splitting defined by $S=\mathbb{Z} a_{1} \oplus \mathbb{Z} b_{1}$ with

$$
\left[a_{1}, b_{1}\right]=\operatorname{Tr}_{\mathbb{Q}}^{K}\left(a_{1} \wedge b_{1}\right)=1 .
$$

Positivity of $S$ means that the determinant $a_{1} \wedge b_{1}$ is a totally positive element of $\mathcal{O}_{D}^{\vee}$. But there are only two such elements with trace one, namely $x$ and $x^{\prime}$ where $x=\epsilon / \sqrt{5}$.

Assume $a_{1} \wedge b_{1}=x$. We claim $S^{\perp}=\epsilon^{-1} S$. To see this, one simply checks that the vectors $\left(a_{2}, b_{2}\right)=\epsilon^{-1}\left(a_{1}, b_{1}\right)$ satisfy $a_{2}, b_{2}$ lie in $S^{\perp}$ and satisfy

$$
\left[a_{2}, b_{2}\right]=\operatorname{Tr}\left(\epsilon^{-2} x\right)=\operatorname{Tr}\left(x^{\prime}\right)=1 .
$$

The case $a_{1} \wedge b_{1}=x^{\prime}$ is similar. 
The following are direct consequences, which could also be verified using the general results referred to in $\S 6$.

1. The nodal curve $P_{D}$ has only one component.

Indeed, for any two splittings $S_{1} \oplus \epsilon S_{1}$ and $S_{2} \oplus \epsilon S_{2}$, any symplectic isomorphism $S_{1} \cong S_{2}$ extends uniquely to an $\mathcal{O}_{D}$-linear automorphism of $L_{D}$.

2. Taking $S_{1}=S_{2}$, we see for any $P \in P\left(L_{D}\right)$ the stabilizer $\mathrm{SL}_{2}\left(\mathcal{O}_{D}\right)^{P}$ is isomorphic to $\mathrm{SL}_{2}(\mathbb{Z})$.

3. The Weierstrass curve $W_{D}$ has only one component, with a unique cusp, since cusps correspond to splittings. We will see a concrete description of $W_{D}$ below.

The golden form. For $D=5$, the standard positive splitting is given by $L_{D}=S \oplus \epsilon^{-1} S$ with

$$
S=\mathbb{Z} \epsilon \oplus \mathbb{Z} i
$$

The form $W_{0}=X_{S}(1)$ corresponds to the symmetric $L$-shaped polygon of Figure 9. Its stabilizer is the well-known Veech group

$$
\Gamma=\mathrm{SL}_{2}\left(\mathcal{O}_{D}\right)^{W_{0}}=\left\langle\left(\begin{array}{cc}
0 & 1 \\
-1 & 0
\end{array}\right),\left(\begin{array}{ll}
1 & \epsilon \\
0 & 1
\end{array}\right)\right\rangle,
$$

which is a $(2,5, \infty)$-triangle group; cf. [V].

Enumerating zeros. Since the Weierstrass curve $W_{D} \cong \mathbb{H} / \Gamma$ has only one cusp, by Proposition 6.3 we have

$$
W\left(L_{D}\right)=\mathrm{AN}_{2}\left(\mathcal{O}_{D}\right) \cdot W_{0} .
$$

In fact, since $\left(\begin{array}{ll}1 & \epsilon \\ 0 & 1\end{array}\right) \cdot W_{0}=W_{0}$, every point in $W\left(L_{D}\right)$ can be expressed uniquely in the form

$$
W_{n, k}=\left(\begin{array}{cc}
\epsilon^{-n} & 0 \\
0 & \epsilon^{n}
\end{array}\right)\left(\begin{array}{cc}
1 & k \\
0 & 1
\end{array}\right) \cdot W_{0}
$$

for some $n, k \in \mathbb{Z}$. We note that the generators of $\mathrm{AN}_{2}\left(\mathcal{O}_{D}\right)$ act on these indices by

$$
\left(\begin{array}{cc}
\epsilon^{-1} & 0 \\
0 & \epsilon
\end{array}\right) \cdot W_{n, k}=W_{n+1, k} \text { and }\left(\begin{array}{cc}
1 & \epsilon \\
0 & 1
\end{array}\right) \cdot W_{n, k}=W_{n, k+F_{2 n}}
$$


Here $F_{n}=(\ldots, 2,-1,1,0,1,1,2,3,5, \ldots)$ is the bi-infinite sequence of Fibonacci numbers, defined by the conditions $F_{n} \in \mathbb{Z}$ and

$$
\epsilon^{n}=F_{n-1}+\epsilon F_{n} .
$$

For fixed $n$, the zeros $W_{n, k}$ appear consecutively along the level set $Y=$ $\epsilon^{n+1}$, and satisfy

$$
\operatorname{Re} \tau_{2}\left(W_{n, k}\right)>0 \text { whenever } k>0 \text {. }
$$

To see this last inequality, use the fact that $W_{0,1}=f_{\gamma}\left(W_{0}\right)$ where $\gamma=$ $\left(\begin{array}{cc}1 & -1 / \epsilon \\ 0 & 1\end{array}\right)$. Since $D f_{\gamma}=\gamma$, this map is a shear on the strip $\epsilon<Y<\epsilon^{2}$. It fixes $W_{1,0}$ on the upper boundary, so it shifts the lower boundary to the right. Since the level sets of $Y$ are symmetric about the imaginary axis, this implies (8.3).

We remark again that the only value of $\tau_{2}\left(W_{n, k}\right)$ known in closed form is the case $\tau_{2}\left(W_{0,0}\right)=\sqrt{-5}$.

Enumerating poles. Let $P_{0}=X_{S}(0)$ be the pole attached to $W_{0}$ by a segment of $\mathcal{F}\left(L_{D}\right)$. Its stabilizer

$$
\operatorname{SL}\left(\mathcal{O}_{D}\right)^{P_{0}}=\left\langle\left(\begin{array}{cc}
0 & \epsilon \\
-\epsilon^{-1} & 0
\end{array}\right),\left(\begin{array}{ll}
1 & \epsilon \\
0 & 1
\end{array}\right)\right\rangle
$$

is conjugate to $\mathrm{SL}_{2}(\mathbb{Z})$.

Define $P_{n, k}$ by replacing $W_{0}$ with $P_{0}$ in (8.1). These give all the poles of $q\left(L_{D}\right)$ in $\mathcal{A}_{0}\left(L_{D}\right)$, since each is attached to a zero by a segment of $\mathcal{F}\left(L_{D}\right)$. Note that the segments $\left[W_{0}, P_{0}\right]$ and $\left[W_{0}, W_{0,1}\right]$ on the leaf $Y=\epsilon$ have $|q|$-lengths 1 and $\epsilon^{-1}$ respectively.

Let $\tau_{1}=\sqrt{-D}$ as usual. Every $P \in P\left(L_{D}\right)$ satisfies $\tau_{2}(P)=U\left(\tau_{1}\right)$ for some $U$ given by equation (5.2). We have $a=0$ in (5.2) when $P \in \mathcal{A}_{0}\left(L_{D}\right)$, and we easily find:

$$
\tau_{2}\left(P_{n, k}\right)=\epsilon^{4 n-2} \tau_{1}+5 k \epsilon^{2 n-1} .
$$

In particular, we have $\tau_{2}\left(P_{0}\right)=i \sqrt{5} / \epsilon^{2}$.

We also have $a \neq 0$ for all $P \in P_{f}\left(L_{D}\right), f>0$, which yields bound

$$
\operatorname{Im} \tau_{2}(P) \leq \frac{1}{a^{2} \operatorname{Im} \tau_{1}} \leq \frac{1}{\sqrt{D}}
$$

This bound is consistent with the fact that $\mathcal{A}_{0}(L)$ contains a halfplane (Corollary 7.4). 


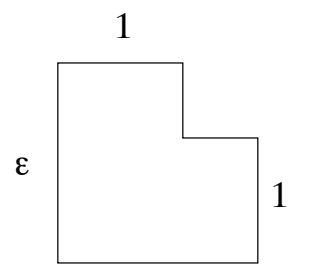

$\varepsilon$

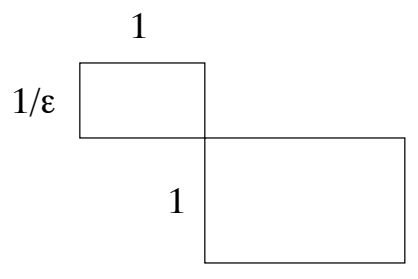

$\varepsilon$

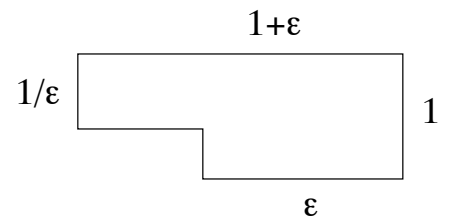

Figure 10. A path along the imaginary axis is obtained by sliding a $1 \times(1 / \epsilon)$ rectangle first horizontally, then vertically.

Overview. An overview of the geometry of $\mathcal{A}\left(L_{D}\right)$, showing the zeros and poles of $q\left(L_{D}\right)$ in $\tau_{2}$-coordinates, is given in Figure 4 .

The imaginary axis. For further orientation, we note that the imaginary axis in $\tau_{2}$-coordinates gives a path through the zeros $W_{n, 0}$ and the poles $P_{n, 0}$. This path runs alternately along the vertical and horizontal foliations of $q\left(L_{D}\right)$. The forms $(X, \omega)$ occurring along two such segments are indicated in Figure 10.

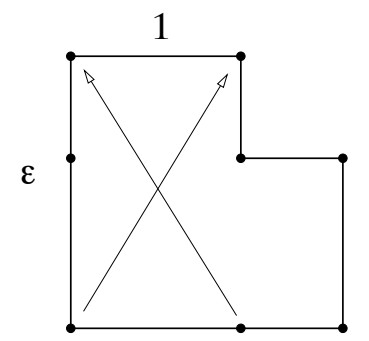

Figure 11. The two types of saddle connections of height $\epsilon$ in the golden table.

Accessible points. We conclude this discussion by showing:

Proposition 8.2 The points of $\mathcal{A}_{f}\left(L_{D}\right) \subset \partial \mathcal{A}_{0}(L)$ are accessible iff $f=1$.

Proof. There are exactly two saddle connections of height $\epsilon$ in $W_{0}$, up to the action of $\mathrm{AN}_{2}\left(\mathcal{O}_{D}\right)^{W_{0}}$ (see Figure 11). One can check that these saddle connection represent the two points in $P_{1}\left(L_{D}\right) / \mathrm{AN}_{2}\left(\mathcal{O}_{D}\right)$; the result then follows from the proof of Theorem 1.9 give in $\S 7$. 


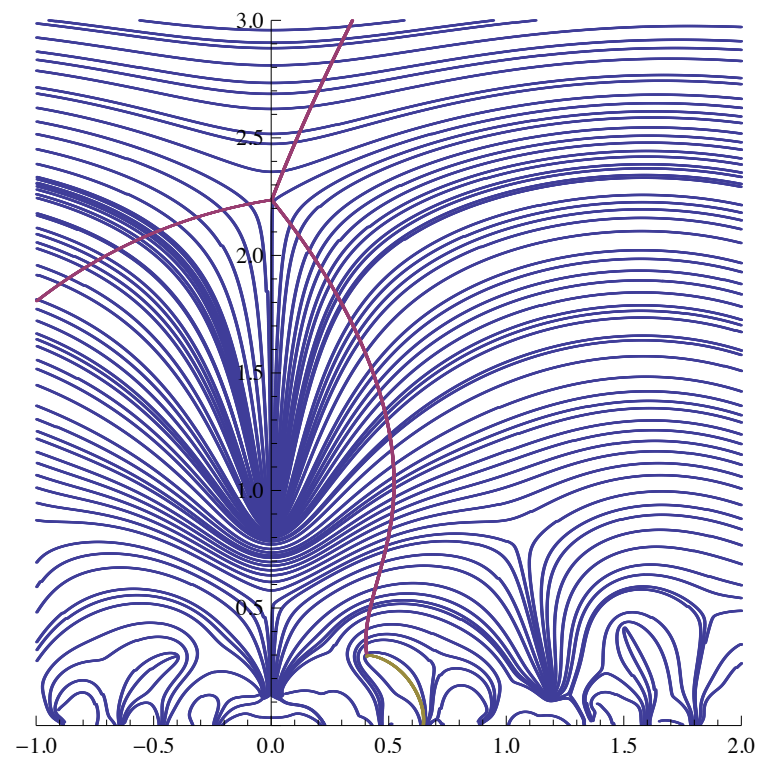

Figure 12. A geodesic in $\mathcal{A}_{0}\left(L_{D}\right)$ connecting $W_{0}$ to a pole in $\mathcal{P}_{1}\left(L_{D}\right)$.

Example. Let $U=\left(\begin{array}{cc}\epsilon^{-1} & 0 \\ 1 & \epsilon\end{array}\right)$. One of the accessible poles $P \in \partial \mathcal{A}_{0}\left(L_{D}\right)$ is given by $\tau_{2}(P)=U\left(\tau_{1}\right)=(1+i) \sqrt{5} /((4+i)+\sqrt{5}) \approx 0.4056+0.2936 i$. This pole corresponds to the symplectic splitting $L_{D}=S \oplus \epsilon S$, where $S=$ $\mathbb{Z}(i / \epsilon) \oplus \mathbb{Z}(1+i)$, as can be read off from Figure 11. A geodesic in $\mathcal{A}_{0}\left(L_{D}\right)$ connecting $W_{0}$ to $P$ is shown in Figure 12 .

\section{$9 \quad$ A guide to fake pentagons}

Let $Q \subset \mathbb{C}$ be a pentagon with unit sides, resting on the interval $[0,1] \subset$ $\mathbb{R}$, and let $Q^{*}$ be its reflection through the real axis. Let $\left(X_{0}, \omega_{0}\right)$ be the pentagon form, obtained from $\left(Q \cup Q^{*}, d z\right)$ by identifying parallel sides. The vertices of $Q \cup Q^{*}$ give the single zero of $\omega_{0}$ on $X_{0}$.

Let $\zeta_{5}=\exp (2 \pi i / 5)$ and let $\tau_{1}=\gamma \zeta_{5} / \sqrt{D}$. It is readily checked that the periods of the pentagon form give the polarized module

$$
\operatorname{Per}\left(X_{0}, \omega_{0}\right)=\mathcal{O}_{D} \oplus \mathcal{O}_{D}^{\vee} \tau_{1}=\mathbb{Z}\left[\zeta_{5}\right],
$$

satisfying $\left[1, \zeta_{5}\right]=1$. As in $[\operatorname{Mc} 5, \S 9]$, we say $(X, \omega) \in \Omega \mathcal{M}_{g}$ is a fake pentagon if:

1. $\omega$ has a double zero; 
2. $\operatorname{Per}(\omega)=\operatorname{Per}\left(\omega_{0}\right)$, respecting the symplectic form; and yet

3. $(X, \omega) \neq\left(X_{0}, \omega_{0}\right)$.

All fake pentagons are also eigenforms for $\mathcal{O}_{D}$. The existence of infinitely many fake pentagons follows from properties of foliations on Hilbert modular surfaces $[$ Mc5, $\S 10]$. In fact, using the enumeration of the zeros of $W\left(L_{D}\right)$, these forms can be explicitly described as follows.

Theorem 9.1 Every fake pentagon can be expressed uniquely in the form $(X, \omega)=A \cdot W_{k, n}$, where

$$
A=\left(\begin{array}{cc}
\epsilon & \operatorname{Re}\left(1+\zeta_{5}\right) \\
0 & \operatorname{Im}\left(1+\zeta_{5}\right)
\end{array}\right) \in \mathrm{GL}_{2}(\mathbb{R})
$$

and $(0,0) \neq(k, n) \in \mathbb{Z}^{2}$.

The regular pentagon is distinguished by the fact that $X_{0}$ also has a symmetry of order 5 with $\omega_{0}$ as an eigenform.

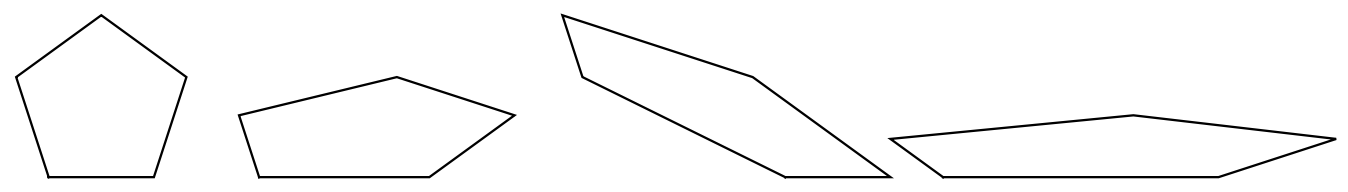

Figure 13. The regular pentagon and several impostors.

\section{Class numbers}

In this section we establish Theorem 1.10, in the following form.

Theorem 10.1 For $D=5$ and $f>0$ we have a natural bijection

$$
P_{f}\left(L_{D}\right) / \mathrm{AN}_{2}\left(\mathcal{O}_{D}\right) \cong \operatorname{Pic}^{+} \mathcal{O}_{f^{2} D}
$$

In particular, the number of different types of splittings of $L_{D}$ of degree $f$ is given by the strict class number $h^{+}\left(f^{2} D\right)$. 
This map depends on the choice of an orientation for $K$ as a vector space over $\mathbb{Q}$.

We will also relate these class numbers to the behavior of Teichmüller rays.

Ideals and conductors. We begin with generalities. Let $K$ be a real quadratic field of discriminant $D>0$. We may identify the ring of integers in $K$ with the real quadratic order $\mathcal{O}_{D}$. The subrings of finite index in $\mathcal{O}_{D}$ are given by the orders

$$
\mathcal{O}_{f^{2} D}=\mathbb{Z}+f \mathcal{O}_{D}
$$

$f \geq 1$. A fractional ideal is an additive subgroup $I \subset K$ isomorphic to $\mathbb{Z}^{2}$. The conductor of $I$ is the unique $f \geq 1$ such that

$$
\mathcal{O}_{f^{2} D}=\left\{x \in \mathcal{O}_{D}: x I \subset I\right\} .
$$

A special property of quadratic fields is that $I$ is invertible over $\mathcal{O}_{f^{2} D}$. The class group $\mathrm{Pic} \mathcal{O}_{f^{2} D}$ consists of the fractional ideals with conductor $f$ modulo principle ideals.

Lemma 10.2 Let $I \subset \mathcal{O}_{D}$ be a submodule of index $f$ such that $\mathcal{O}_{D} \cdot I=\mathcal{O}_{D}$. Then $f$ is the conductor of $I$.

Proof. We first note that $\mathcal{O}_{D} / I$ must be isomorphic to $\mathbb{Z} / f$ as an additive group; otherwise $I \subset n \mathcal{O}_{D}$ for some $n>1$, in which case $\mathcal{O}_{D} \cdot I \neq \mathcal{O}_{D}$. Let $e$ be the conductor of $I$. Since $\mathcal{O}_{e^{2} D} \cdot I \subset I$, we have $e \mathcal{O}_{D} \cdot I=e \mathcal{O}_{D} \subset I$, and thus $f \mid e$. On the other hand, since $\left[\mathcal{O}_{D}: I\right]=f$ we have $f \mathcal{O}_{D} \cdot I=$ $f \mathcal{O}_{D} \subset I$, and thus $e \mid f$.

Remark. More generally, the conductor of any fractional ideal satisfies $f=\left[\mathcal{O}_{D} I: I\right]$.

Class numbers. An explicit formula for the class number $h\left(f^{2} D\right)=$ $\left|\operatorname{Pic} \mathcal{O}_{f^{2} D}\right|$, in the case $D=5$, can be given in terms of the Legendre symbol and the Fibonacci sequence $F_{n}$ defined in equation (8.2); namely we have

$$
h\left(f^{2} D\right)=\frac{f}{\min \left(n: f \mid F_{n}\right)} \prod_{p \mid f}\left(1-\left(\frac{p}{5}\right) \frac{1}{p}\right) .
$$

This can be seen using [Lang, Ch. 8.1, Thm. 7] and the fact that $\mathbb{Z}\left[\epsilon^{n}\right]=$ $\mathcal{O}_{F_{n}^{2} D}$

If we only take the quotient by the principal ideals $(k)$ with $N(k)>0$, we obtain the strict class group $\mathrm{Pic}^{+} \mathcal{O}_{f^{2} D}$, whose order will be denoted $h^{+}\left(f^{2} D\right)$. 
These two types of class groups are identical if there is a unit of norm -1 in $\mathcal{O}_{f^{2} D}$; otherwise, we have $h^{+}\left(f^{2} D\right)=2 h\left(f^{2} D\right)$. For $D=5$, we have $h\left(f^{2} D\right)=h^{+}\left(f^{2} D\right)$ iff $f \mid F_{n}$ for some odd value of $n$. The first few values are shown in Table 14 .

\begin{tabular}{|c|cccccccccccc|}
\hline$f$ & 1 & 2 & 3 & 4 & 5 & 6 & 7 & 8 & 9 & 10 & 11 & 12 \\
\hline$h^{+}\left(f^{2} D\right)$ & 1 & 1 & 2 & 2 & 1 & 2 & 2 & 4 & 2 & 1 & 2 & 4 \\
\hline \hline$f$ & 13 & 14 & 15 & 16 & 17 & 18 & 19 & 20 & 21 & 22 & 23 & 24 \\
\hline$h^{+}\left(f^{2} D\right)$ & 2 & 2 & 2 & 4 & 2 & 6 & 2 & 2 & 8 & 2 & 2 & 8 \\
\hline
\end{tabular}

Table 14. Strict class numbers of orders of conductor $f$, for $D=5$.

If we fix an orientation for $K \cong \mathbb{Q}^{2}$, we may identify the strict class group with the set of isomorphism classes of oriented invertible $\mathcal{O}_{f^{2} D^{-m o d u l e s}}$

Proof of Theorem 10.1. By Proposition 8.1, points $Z \in P_{f}\left(L_{D}\right)$ corresponds bijectively to splittings of the form $L_{D}=S \oplus S^{\perp}$ such that $S^{\perp}=\epsilon S$ and $\left[\mathcal{O}_{D}: I\right]=f$, where $I=\operatorname{Im}(S)$. Since $\mathcal{O}_{D} I=\mathcal{O}_{D}$, the preceding Lemma shows the conductor of $I$ is $f$. The symplectic form on $S$ determines an orientation of $I$, and thus we have a map

$$
\phi: P_{f}\left(L_{D}\right) \rightarrow \operatorname{Pic}^{+} \mathcal{O}_{f^{2} D}
$$

given by $\phi(Z)=[\operatorname{Im}(S)]$.

Since $\mathcal{O}_{D}$ has strict class number one, any oriented fractional ideal $I=$ $\mathbb{Z} a \oplus \mathbb{Z} b$ of conductor $f$ can be scaled so that $\mathcal{O}_{D} I=\mathcal{O}_{D}$. Then ad$b c=1$ for some $c, d \in \mathcal{O}_{D}$. This gives an element $\gamma \in \mathrm{SL}_{2}\left(\mathcal{O}_{D}\right)$ such that $\phi\left(\gamma\left(P_{0}\right)\right)=[I]$, and thus $\phi$ is surjective.

Next we observe that $\phi$ is constant along the orbits of $\operatorname{AN}_{2}\left(\mathcal{O}_{D}\right)$. Indeed, if $\gamma \in \mathrm{AN}_{2}\left(\mathcal{O}_{D}\right)$ then $\operatorname{Im}(\gamma(S))=\epsilon^{n} \operatorname{Im}(S)$ for some $n \in \mathbb{Z}$.

Finally suppose $\phi\left(Z_{1}\right)=\phi\left(Z_{2}\right)$. Then the corresponding splittings $S_{1} \oplus$ $S_{1}^{\perp}$ and $S_{2} \oplus S_{2}^{\perp}$ determine the same oriented ideal class. This means that, after applying the action of the diagonal subgroup of $\mathrm{AN}_{2}\left(\mathcal{O}_{D}\right)$ to one of them, we can arrange that $\operatorname{Im}\left(S_{1}\right)=\operatorname{Im}\left(S_{2}\right)$ as oriented fractional ideals. Using the fact that $S_{i}^{\perp}=\epsilon S_{i}$, we find there is a unique $\gamma \in \mathrm{SL}_{2}\left(\mathcal{O}_{D}\right)$ inducing the isomorphism $S_{1} \cong \operatorname{Im}\left(S_{1}\right) \cong \operatorname{Im}\left(S_{2}\right) \cong S_{2}$. Since $\operatorname{Im} \gamma(s)=\operatorname{Im}(s)$ for all $s \in S_{1}$, we have $\gamma \in \mathrm{N}_{2}\left(\mathcal{O}_{D}\right)$, and thus $Z_{1}$ and $Z_{2}$ lie in the same orbit of $\mathrm{AN}_{2}\left(\mathcal{O}_{D}\right)$. 
Double cosets. Here is another perspective on the result above. When $D=5$ the group $\mathrm{SL}_{2}\left(\mathcal{O}_{D}\right)$ acts transitively on $P\left(L_{D}\right)$ with point stabilizers isomorphic to $\mathrm{SL}_{2}(\mathbb{Z})$. On the other hand, since $\mathcal{O}_{D}$ has class number one, the group $\mathrm{SL}_{2}\left(\mathcal{O}_{D}\right)$ acts transitively on $\mathbb{P}^{1}(K)$ with $\mathrm{AN}_{2}\left(\mathcal{O}_{D}\right)$ the stabilizer of infinity. Thus we have a correspondence:

$$
\begin{aligned}
\mathrm{AN}_{2}\left(\mathcal{O}_{D}\right) \backslash P\left(L_{D}\right) & \Longleftrightarrow \mathrm{AN}_{2}\left(\mathcal{O}_{D}\right) \backslash \mathrm{SL}_{2}\left(\mathcal{O}_{D}\right) / \mathrm{SL}_{2}(\mathbb{Z}) \\
& \Longleftrightarrow \mathbb{P}^{1}(K) / \mathrm{SL}_{2}(\mathbb{Z}) .
\end{aligned}
$$

Any point in $\mathbb{P}^{1}(K)$ can be given in homogeneous coordinates as $[a: b]$ where $a, b \in \mathcal{O}_{D}$ are relatively prime; then the conductor $f=\left|a b^{\prime}-a^{\prime} b\right| / \sqrt{D}$ of the ideal $\mathbb{Z} a \oplus \mathbb{Z} b$ is constant along the orbits on the right, and corresponds to the degree of the splittings on the left. It is immediate that the number of orbits on the right with a given conductor is $h^{+}\left(f^{2} D\right)$.

A partial order on ideals classes. The leaves of $\mathcal{F}\left(L_{D}\right)$ have a natural partial ordering given by $\mathcal{F}_{1} \leq \mathcal{F}_{2}$ if $\mathcal{F}_{2} \subset \overline{\mathrm{AN}_{2}\left(\mathcal{O}_{D}\right) \cdot \mathcal{F}_{1}}$. This ordering is compatible with the ordering by upper degree, and it descends to an ordering on the set of ideals classes

$$
\mathrm{Cl}(K)=\bigcup_{f>0} \operatorname{Pic}\left(\mathcal{O}_{f^{2} D}\right) .
$$

It would be interesting to describe this ordering directly in terms of the structure of $\mathrm{Cl}(K)$.

Escaping Teichmüller rays. For another perspective on these matters, let $\left(X_{0}, \omega_{0}\right.$ be a form in $\mathcal{A}_{f}\left(L_{D}\right)-P_{f}\left(L_{D}\right)$, and consider the Teichmüller ray $\left[X_{t}\right]$ in $\mathcal{M}_{2}$ obtained by projection the forms

$$
\left(\begin{array}{cc}
e^{-t} & 0 \\
0 & e^{t}
\end{array}\right) \cdot\left(X_{0}, \omega_{0}\right) \in \Omega \mathcal{M}_{2}, \quad t \geq 0
$$

to moduli space. The leaves of the horizontal foliation $\mathcal{F}\left(\omega_{0}\right)$ shrink to zero along this ray.

If $f=0$, then these leaves are closed and $\left[X_{t}\right]$ converges to a stable curve in $\partial \mathcal{M}_{2}$ consisting of one or two components of genus zero. In particular, $\left[\operatorname{Jac}\left(X_{t}\right)\right]$ goes to infinity in the Hilbert modular surface for $\mathcal{O}_{D}$.

If $f>0$, on the other hand, then $\left(X_{0}, \omega_{0}\right)$ splits as the connected sum of a pair of complex tori with irrational foliations, and $X_{t}$ spirals towards a closed geodesic $\gamma$ on a modular curve inside $\mathcal{M}_{1} \times \mathcal{M}_{1} \subset \partial \mathcal{M}_{2}$. In this case $\left[\operatorname{Jac}\left(X_{t}\right)\right]$ remains bounded in the Hilbert modular surface, and the hyperbolic length of $\gamma$ is the $\log$ of a unit in $\mathcal{O}_{D}$. For $D=5$, the modular curve is simply $\mathbb{H} / \mathrm{SL}_{2}(\mathbb{Z})$, and the oriented geodesic $\gamma$ determines an ideal class in $\mathrm{Pic}^{+} \mathcal{O}_{f^{2} D}$. 


\section{Snow falling on cedars}

In this section we describe an explicit path in $\mathcal{A}_{0}(L)$ joining together the points $W_{0, k}, k \in \mathbb{Z}$, and use it to construct a family of interval exchange maps $F_{t}(x)$ whose bifurcation set is homeomorphic to $\omega^{\omega}$. This family gives an indication of the complexity of $\partial \mathcal{A}_{0}(L)$, and the way bifurcations and periodicity combine to render $\mathcal{A}(L)$ complete.

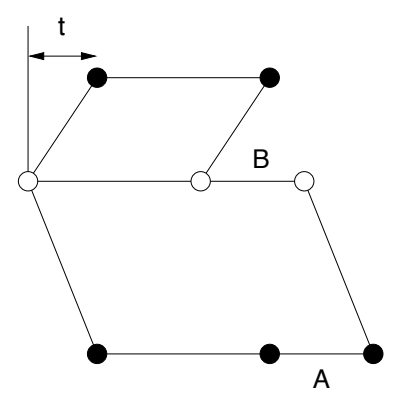

Figure 15. The form $\left(X_{t}, \omega_{t}\right)$ is obtained from the symmetric form $W_{0}$ by shearing horizontally, preserving the absolute periods. Each point $(x, 0) \in A$ is glued to the point $\left(x^{\prime}, 1\right) \in B$ with $x^{\prime}=x \bmod |A|$.

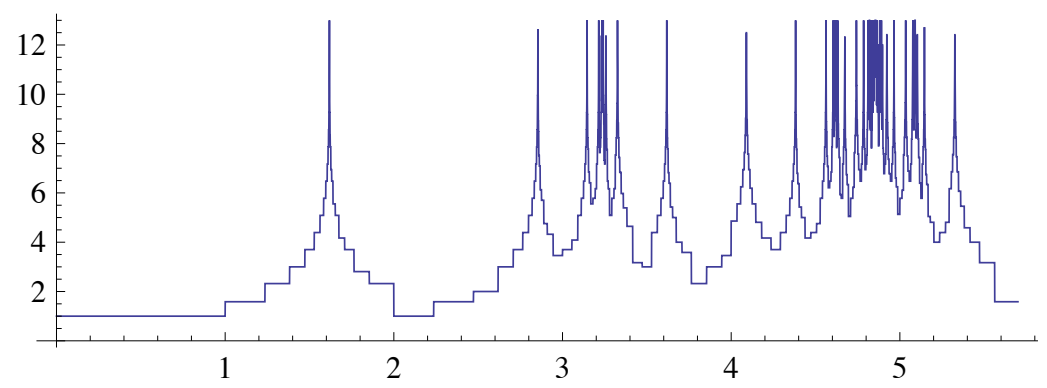

Figure 16. Spikes in the period of $F_{t}(x)$ occur for $t$ in a closed, countable set $B$ homeomorphic to $\omega^{\omega}$.

A path through the zeros. Let $\left(X_{0}, \omega_{0}\right)=W_{0}$, presented as the quotient of a symmetric $L$-shaped polygon with edges of lengths 1 and $\epsilon$ as in Figure 11. By shearing the two rectangles form $\left(X_{0}, \omega_{0}\right)$ independently, we obtain the family of forms $\left(X_{t}, \omega_{t}\right)$ in $\mathcal{A}\left(L_{D}\right)$ as shown in Figure 15 . (The same general construction was used in the proof of Proposition 4.3.) Since the 


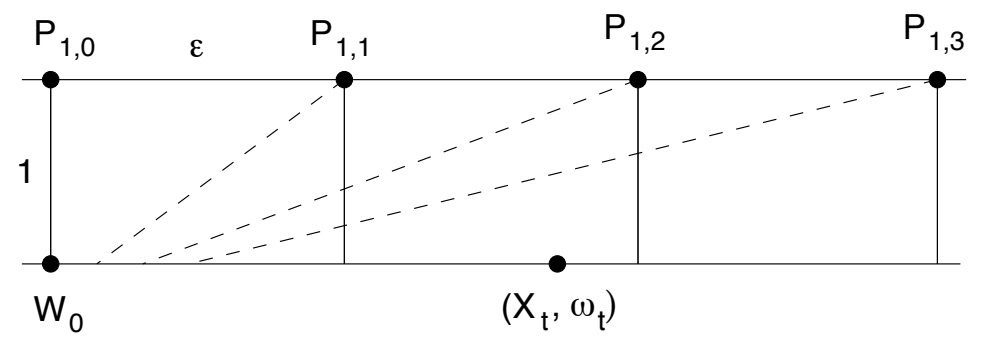

Figure 17. Stable and unstable manifolds which cross.

zeros of $\omega_{t}$ only move horizontally, this family gives a path along the leaf of $\mathcal{F}\left(L_{D}\right)$ through $W_{0}$. In fact the forms $\left(X_{t}, \omega_{t}\right)$ move at unit speed along the upper edge of the leaf through $W_{0}$, passing through $W_{0, k}$ at time $t=k / \epsilon$.

Now consider the vertical foliation $\mathcal{F}\left(i \omega_{t}\right)$ of $X_{t}$. Its first return map to the bottom edge in Figure 15 gives an interval exchange transformation

$$
F_{t}:[0, \epsilon] \rightarrow[0, \epsilon]
$$

which rotates the subintervals $[0,1]$ and $[1, \epsilon]$ each by $+t$, and then rotates the full interval $[0, \epsilon]$ by $-t$.

Theorem 11.1 The bifurcation set

$$
B=\left\{t \in \mathbb{R}: \mathcal{F}\left(i \omega_{t}\right) \text { is not periodic }\right\}
$$

is homeomorphic to $\omega^{\omega}$. In fact for each $k>0$, the derived set $B^{(k-1)}$ contains $k \epsilon$.

(Here $B^{0}=B$ and $B^{(k+1)}=\partial B^{(k)}$.) The set $B$ consists of the points the period $N_{t}$ of $F_{t}$ becomes infinite. The graph of $\log _{2}\left(N_{t}\right)$ is shown in Figure 16.

Proof. The region $\epsilon<Y<\epsilon^{2}$ is an infinite strip of height one in the $|q|$-metric. If we take its closure and unfold the upper edge, we obtain the region shown in Figure 17. The form $\left(X_{t}, \omega_{t}\right)$ moves at unit speed along the lower edge. When $t=k \epsilon$, it encounters the vertical leaf through $P_{1, k}$. Thus these values of $t$ lie in the bifurcation set.

For each $k>0$ there is a hyperbolic element $\gamma_{k} \in \mathrm{SL}_{2}\left(\mathcal{O}_{D}\right)$ stabilizing $P_{1, k}$ and expanding the vertical leaf on which it lies. The contracting direction of $\gamma_{k}$ gives a stable manifold through $P_{1, k}$, which is shown as a dotted line in Figure 17. 
We claim the stable manifold through $P_{1, k}$ crosses the vertical leaf through $P_{1, k-1}$. To verify this, one can one use the fact that the stabilizer of $P_{1, k}$ is given by $A_{k} \mathrm{SL}_{2}(\mathbb{Z}) A_{k}^{-1}$, where $A_{k}=\left(\begin{array}{cc}1 & k / \epsilon \\ 0 & \epsilon\end{array}\right)$. The stable manifold through $P_{1, k}$ is parallel to the vector $A_{k}\left(A_{k}^{-1}\left(\begin{array}{l}0 \\ 1\end{array}\right)\right)^{\prime}$, and we find it crosses the path $\left(X_{t}, \omega_{t}\right)$ at $t=k / \epsilon^{3}$.

By the mechanism illustrated in Figure 3, the intersection of stable and unstable manifolds results in copies of the vertical leaf through $P_{1, k-1}$ accumulating on the vertical leaf through $P_{1, k}$. It follows easily by induction that $k \epsilon \in B^{(k-1)}$. This shows $B$ contains a homeomorphic copy of the countable ordinal $\omega^{\omega}$. It can be no more complex than this ordinal, by equation (4.3).

The additional fact that $k \epsilon \notin B^{(k)}$ can be established using [Mc7, Theorem 10.1].

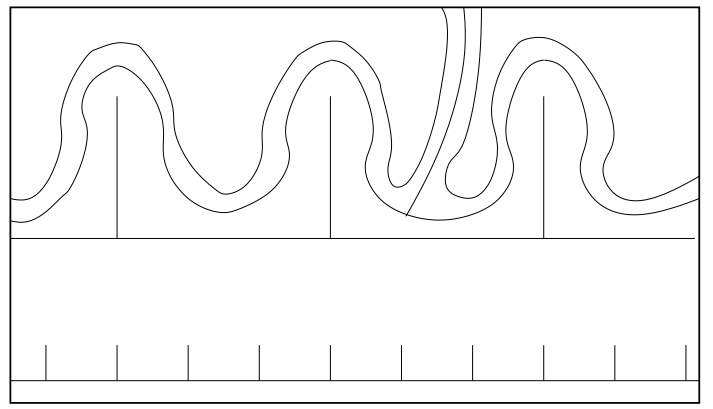

Figure 18. Cedars and snow.

Winters with diminishing snowfall. We conclude by relating these bifurcations to the completeness of $\mathcal{A}\left(L_{D}\right)$.

We have seen that $\mathcal{A}_{0}\left(L_{D}\right)$ is a stacked sequence of horizontal strips, each mapping to an interval under the height function $Y: \mathcal{A}_{0}\left(L_{D}\right) \rightarrow \mathbb{R}_{+}$. One can think of these strips as layers of snow, and $Y$ as depth beneath the snow. We wish to picture the region $0 \leq Y \leq \epsilon$.

Think of the set $Y=\epsilon$ as a line of level ground, along which a sequence of cedars $\left[W_{0, k}, P_{0, k}\right]$ have grown to height 1 (Figure 18). During the winter these trees are buried under a layer of snow of thickness $1 / \epsilon$. The next spring taller cedars grow, farther apart, and then the next winter they are buried under a thinner layer of snow. The process continues through countably many seasons until we reach the top of the snow at depth $Y=0$. 
In fact with each new season the trees get higher by a factor of $\epsilon>1$, while the snow gets thinner by the same factor. Thus the height of the cedars tends to infinity, while the total depth of the snow, after infinitely many seasons, is just $\epsilon$. It is therefore possible to burrow out of the snow by traveling only a finite distance; that is, $\mathcal{A}_{0}\left(L_{D}\right)$ is incomplete.

It becomes complete, however, when we add in the countably many half-infinite leaves of $\mathcal{F}\left(L_{D}\right)$ contained in $\bigcup_{f>0} \mathcal{A}_{f}\left(L_{D}\right)$. These leaves can be visualized as fissures where the snowfall, in infinitely many layers, folds together to meet itself. An explorer attempting to burrow out of $\mathcal{A}_{0}\left(L_{D}\right)$ encounters a countable cascade of such fissures, and finds himself descending back into the snow. One can think of Figure 16 as a record of the explorer's journey, with spikes occurring each time a fissure is crossed.

\section{References}

[Ba] M. Bainbridge. Euler characteristics of Teichmüller curves in genus two. Geom. Topol. 11(2007), 1887-2073.

[BL] C. Birkenhake and H. Lange. Complex Abelian Varieties. SpringerVerlag, 1992.

[Bol] A. A. Bolibruch. Inverse monodromy problems of the analytic theory of differential equations. In Mathematical Events of the Twentieth Century, pages 49-74. Springer, 2006.

[CW] K. Calta and K. Wortman. On unipotent flows on $\mathcal{H}(1,1)$. Erg. Th. E Dynam. Sys. 30(2010), 379-398.

[vG] G. van der Geer. Hilbert Modular Surfaces. Springer-Verlag, 1987.

[Kap] M. Kapovich. Periods of abelian differentials and dynamics. Preprint, 2000.

[KZ] M. Kontsevich and A. Zorich. Connected components of the moduli spaces of Abelian differentials with prescribed singularities. Invent. math. 153(2003), 631-678.

[Lang] S. Lang. Elliptic Functions. Springer-Verlag, 1987.

[La] E. Lanneau. Hyperelliptic components of the moduli spaces of quadratic differentials with prescribed singularities. Comment. Math. Helv. 79(2004), 471-501. 
[Mc1] C. McMullen. Billiards and Teichmüller curves on Hilbert modular surfaces. J. Amer. Math. Soc. 16(2003), 857-885.

[Mc2] C. McMullen. Teichmüller curves in genus two: Discriminant and spin. Math. Ann. 333(2005), 87-130.

[Mc3] C. McMullen. Teichmüller curves in genus two: Torsion divisors and ratios of sines. Invent. math. 165(2006), 651-672.

[Mc4] C. McMullen. Dynamics of $\mathrm{SL}_{2}(\mathbf{R})$ over moduli space in genus two. Annals of Math. 165(2007), 397-456.

[Mc5] C. McMullen. Foliations of Hilbert modular surfaces. Amer. J. Math. 129(2007), 183-215.

[Mc6] C. McMullen. Navigating moduli space with complex twists. J. Eur. Math. Soc. 15(2013), 1223-1243.

[Mc7] C. McMullen. Cascades in the dynamics of measured foliations. To appear, Ann. scient. Éc. Norm. Sup.

[Mo] M. Möller. Affine groups of flat surfaces. In A. Papadopoulos, editor, Handbook of Teichmüller Theory, volume II, pages 369-387. Eur. Math. Soc., 2009.

[MZ] M. Möller and D. Zagier. Theta derivatives and Teichmüller curves. In preparation.

[Mu R. Mukamel. Orbifold points on Teichmüller curves and Jacobians with complex multiplication. Preprint, 2012.

[Mum] D. Mumford. Tata Lectures on Theta I. Birkhäuser, 1983.

[Rat] M. Ratner. Interactions between ergodic theory, Lie groups and number theory. In Proceedings of the International Congress of Mathematicians (Zürich, 1994), pages 156-182. Birkhaüser, Basel, 1995.

[Sch] M. Schmoll. Spaces of elliptic differentials. In Algebraic and topological dynamics, volume 385 of Contemp. Math., pages 303-320. Amer. Math. Soc., 2005.

[V] W. Veech. Teichmüller curves in moduli space, Eisenstein series and an application to triangular billiards. Invent. math. 97(1989), $553-583$. 
[Z] A. Zorich. Flat surfaces. In Frontiers in Number Theory, Physics, and Geometry. I, pages 437-583. Springer, 2006.

Mathematics Department

HARVARD UNIVERSITY

1 OXFORD ST

CAMbridge, MA 02138-2901 